\title{
RAMIFIED GALOIS COVERS VIA MONOIDAL FUNCTORS
}

\author{
FABIO TONINI
}

\begin{abstract}
We interpret Galois covers in terms of particular monoidal functors, extending the correspondence between torsors and fiber functors. As applications we characterize tame $G$-covers between normal varieties for finite and étale group schemes and we prove that, if $G$ is a finite, flat and finitely presented nonabelian and linearly reductive group scheme over a ring, then the moduli stack of $G$-covers is reducible.
\end{abstract}

\section{INTRODUCTION}

Let $R$ be a base commutative ring and $G$ be a flat, finite and finitely presented group scheme over $R$. In [Ton13a] I introduced the notion of a ramified Galois cover with group $G$, briefly a $G$-cover, and the stack $G$-Cov of such objects (see 1.2 for details). This stack is algebraic and of finite type over $R$ and contains $\mathrm{B}_{R} G$, the stack of $G$-torsors, as an open substack. If $G$ is diagonalizable, its nice representation theory makes it possible to study $G$-covers in terms of simplified data (collections of invertible sheaves and morphisms between them) and to investigate the geometry of the moduli $G$-Cov (see [Ton13a]).

The general case is much harder, even when $G$ is a constant group over an algebraically closed field of characteristic zero: a direct approach as in the diagonalizable case fails because of the complexity of the representation theory of $G$. Thus in order to handle general $G$-covers one needs a different perspective and Tannaka's duality comes into play. The $G$-torsors are very special $G$-covers and the solution of Tannaka's reconstruction problem asserts that they can be described in terms of particular strong monoidal functors with domain $\operatorname{Loc}^{G} R$, the category of $G$-comodules over $R$ which are projective and finitely generated as $R$-modules. If $\mathcal{X}$ is an algebraic stack, denote by Loc $\mathcal{X}$ (resp. QCoh $\mathcal{X}$ ) the category of locally free of finite rank (resp. quasi-coherent) sheaves on $\mathcal{X}$, so that $\operatorname{Loc}_{R} G \simeq \operatorname{Loc}^{G} R$. When $\mathcal{X}=\operatorname{Spec} A$ we simply write Loc $A$ and $\mathrm{QCoh} A$. The result about $G$-torsors can be stated as follows.

Theorem. ([DM82, Theorem 3.2], [Sch13, Theorem 1.3.2]) Let $\mathrm{SMon}_{R}^{G}$ be the stack over $R$ whose fiber over an $R$-scheme $T$ is the category of R-linear, exact (on short exact sequences) and strong monoidal functors $\operatorname{Loc}^{G} R \longrightarrow \operatorname{Loc} T$. Then the functor

$$
\begin{gathered}
\mathrm{B}_{R} G \stackrel{\Delta}{\longrightarrow} \operatorname{SMon}_{R}^{G} \\
\left(T \stackrel{s}{\longrightarrow} \mathrm{B}_{R} G\right) \longmapsto \\
s_{\mid \operatorname{Loc}^{G} R}^{*}
\end{gathered}
$$

is an equivalence of stacks.

Since a $G$-cover is a "weak" version of a $G$-torsor it is natural to look at a "weak" version of a strong monoidal functor, that is, as the words suggest, a (lax) monoidal functor. This idea has motivated the study in [Ton14] of more general monoidal (and non) functors and this paper is an application of it. We introduce the stack $\operatorname{Mon}_{R}^{G}\left(\operatorname{Mon}_{R, \text { reg }}^{G}\right)$ over $R$ whose fiber over an $R$-scheme $T$ is the groupoid of $R$-linear, exact monoidal functors $\Gamma: \operatorname{Loc}^{G} R \longrightarrow \operatorname{Loc} T$ (such that $\operatorname{rk} \Gamma_{V}=\operatorname{rk} V$ (pointwise) for all $V \in \operatorname{Loc}^{G} R$ ). We also denote by $\operatorname{LAlg}_{R}^{G}$ the stack over 
$R$ whose fiber over an $R$-scheme $T$ is the groupoid of locally free sheaves of algebras on $T$ with an action of $G$, or, alternatively, the stack of covers with an action of $G$. The stack $\operatorname{LAlg}_{R}^{G}$ is algebraic and locally of finite presentation over $R$ and $G$-Cov is an open substack of $\operatorname{LAlg} G$ (see $1.5)$.

Recall that $G$ is linearly reductive over $R$ if the functor of invariants $(-)^{G}: \mathrm{QCoh} \mathrm{B}_{R} G \longrightarrow$ QCoh $R$ is exact. We say that $G$ has a good representation theory over $R$ if it is linearly reductive and there exists a finite collection $I_{G}$ of sheaves in $\operatorname{Loc}^{G} R$ such that for all geometric points (one is enough if $\operatorname{Spec} R$ is connected) Spec $k \longrightarrow \operatorname{Spec} R$ the map $\left(-\otimes_{R} k\right): I_{G} \longrightarrow \operatorname{Loc}^{G} k$ is a bijection onto a collection of representatives of the irreducible representations of $G \times{ }_{R} k$. Examples of groups with a good representation theory are diagonalizable groups and linearly reductive groups over algebraically closed fields. In general we show that any linearly reductive group $G$ over $R$ has fppf locally (étale locally if $G / R$ is étale) a good representation theory (see 1.15).

Theorem A. The map of stacks

$$
\widetilde{\Delta}: G-\operatorname{Cov} \longrightarrow \operatorname{Mon}_{R}^{G},(X \stackrel{f}{\longrightarrow} T) \longmapsto\left(f_{*} \mathcal{O}_{X} \otimes-\right)^{G}
$$

is an open immersion, it extends the equivalence $\Delta: \mathrm{B}_{R} G \longrightarrow \operatorname{SMon}_{R}^{G}$ and takes values in $\operatorname{Mon}_{R, \text { reg }}^{G}$.

If $G$ is linearly reductive over $R$ then $\widetilde{\Delta}$ extends to an equivalence $\widetilde{\Delta}: \operatorname{LAlg}_{R}^{G} \longrightarrow \operatorname{Mon}_{R}^{G}$, namely $\widetilde{\Delta}(\mathscr{A})=(\mathscr{A} \otimes-)^{G}$, the stack $G$-Cov is an open and closed substack of $\operatorname{LAlg}_{R}^{G}$ and, if $G$ has a good representation theory, then $\widetilde{\Delta}(G$-Cov $)=\operatorname{Mon}_{R, \text { reg }}^{G}$.

The equality $\widetilde{\Delta}(G$-Cov $)=\operatorname{Mon}_{R \text {,reg }}^{G}$ is not true in general, even when $G$ is linearly reductive (see 1.8).

We are going to show two applications of the above point of view. The first one is about the geometry of $G$-Cov (see also 3.3 ).

Theorem B. If $G$ is a finite, flat and finitely presented nonabelian linearly reductive group scheme over $R$ then the stack $G$-Cov is reducible.

When $G$ is a diagonalizable group the same result holds except for a few cases when $G$ has low rank (see [Ton13a, Corollary 4.17]). Thus the bad behaviour of the moduli $G$-Cov is still present in the nonabelian setting. Note that the proof of Theorem B does not use and cannot be adapted to show the reducibility of $G$-Cov when $G$ is a diagonalizable group. Moreover it requires the

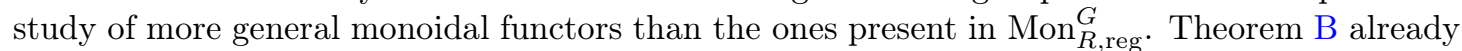
appeared in my Ph.D. thesis [Ton13b], but the proof we present here is slightly different and relies on the following fact: if $H$ is an open and closed subgroup scheme of $G$ the functor

$$
\operatorname{ind}_{H}^{G}: \operatorname{LAlg}_{R}^{H} \longrightarrow \operatorname{LAlg}_{R}^{G}, \mathscr{A} \longmapsto(\mathscr{A} \otimes R[G])^{H}
$$

is well defined, quasi-affine and étale (see 2.1).

The second application is a characterization of $G$-covers of regular in codimension 1 schemes. Let us introduce some notation and definitions in order to explain the result. Let $f: X \longrightarrow T$ be a cover with an action of $G$ on $X$. We denote by $\operatorname{tr}_{f}: f_{*} \mathcal{O}_{X} \longrightarrow \mathcal{O}_{T}$ the trace map, by $\widetilde{\operatorname{tr}}_{f}: f_{*} \mathcal{O}_{X} \longrightarrow\left(f_{*} \mathcal{O}_{X}\right)^{\vee}$ the map $x \longmapsto \operatorname{tr}_{f}(x \cdot-)$ and by $s_{f} \in\left(\operatorname{det} f_{*} \mathcal{O}_{X}\right)^{-2}$ the discriminant section, that is the section obtained by $\operatorname{det} \widetilde{\operatorname{tr}}_{f}$. If $f$ is a $G$-cover with associated monoidal functor $\Omega^{f}=\left(f_{*} \mathcal{O}_{X} \otimes-\right)^{G}: \operatorname{Loc}^{G} R \longrightarrow \operatorname{Loc} T$ and $V \in \operatorname{Loc}^{G} R$ consider

$$
\Omega_{V}^{f} \otimes \Omega_{V^{\vee}}^{f} \longrightarrow \Omega_{V \otimes V^{\vee}}^{f} \longrightarrow \Omega_{R}^{f}=\left(f_{*} \mathcal{O}_{X}\right)^{G}=\mathcal{O}_{T}
$$

where the first map is given by monoidality, while the second is induced by the evaluation $V \otimes V^{\vee} \longrightarrow R$. The morphism above yields a map $\xi_{f, V}: \Omega_{V^{\vee}}^{f} \longrightarrow\left(\Omega_{V}^{f}\right)^{\vee}$ of locally free sheaves 
whose rank coincides with $\operatorname{rk} V$ by Theorem A. Applying the determinant we obtain a section $s_{f, V} \in\left(\operatorname{det} \Omega_{V}^{f} \otimes \operatorname{det} \Omega_{V^{\vee}}^{f}\right)^{-1}$. If $q \in T$ is a point and $V \in \operatorname{Loc}^{G} T$ we denote by $\operatorname{rk}_{q} V$ the rank of $V \otimes \mathcal{O}_{T, q}$ and by $\operatorname{rk}_{q} G$ the rank of $G$ over $q$, that is $\operatorname{rk}_{q} \mathcal{O}_{T}[G]$. The result we will prove is the following.

Theorem C. Let $G$ be a finite and étale group scheme over $R$. Let also $Y$ be an integral and Noetherian $R$-scheme with $\operatorname{dim} Y \geq 1$, and $f: X \longrightarrow Y$ be a cover with an action of $G$ on $X$ over $Y$ and such that $X / G=Y$. Let also $q \in Y$ be a codimension 1 and regular point. Then the following are equivalent:

1) all points of $X$ over $q$ are regular, tame (the ramification index is coprime with char $k(q)$ ) and have separable residue fields.

2) we have $v_{q}\left(s_{f}\right)<\operatorname{rk} f$, where $v_{q}$ denotes the valuation in $q$;

3) there exist an étale neighborhood $U \longrightarrow Y$ with a point $q^{\prime}$ mapping to $q$ and with $G \times U$ constant, subgroups $T \triangleleft H<G \times U$ with $H / T$ cyclic of order coprime with char $k(q)$ and $\operatorname{Spec} \mathscr{B} \in(H / T)-\operatorname{Cov}(U)$ such that $X \times_{Y} U=\operatorname{Spec}\left(\operatorname{ind}_{H}^{G} \mathscr{B}\right), \mathscr{B}_{q^{\prime}}$ is a regular local ring, $H$ is the geometric stabilizer of a codimension 1 point of $X$ over $q, T$ is the geometric stabilizer of a generic point of $X$ and $\operatorname{Spec} \mathscr{B}$ is generically an $(H / T)$-torsor.

If one of the above conditions is satisfied we have that: $f$ is generically a G-torsor if and only if $\operatorname{rk} f=\operatorname{rk} G$ and in this case the geometric stabilizers of the codimension 1 points of $X$ over $q$ are linearly reductive and cyclic and there exists an open subset $V \subseteq Y$ containing $q$ and such that $f_{\mid f^{-1}(V)}: f^{-1}(V) \longrightarrow V$ is a $G$-cover; if $G$ is constant, $G \longrightarrow$ Aut $X$ is injective and the generic fiber of $f: X \longrightarrow Y$ is connected then $\operatorname{rk} f=\operatorname{rk} G$.

If $G$ is linearly reductive and $\operatorname{rk} f=\operatorname{rk} G$ then the above conditions are equivalent to

4) $f \in G$-Cov and for all $V \in \operatorname{Rep}^{G} R$ (resp. $V \in I_{G}$ if $G$ is good) we have $v_{q}\left(s_{f, V}\right) \leq$ $\operatorname{rk}_{q}\left(V / V^{G}\right)$;

5) $f \in G$-Cov and for all $V \in \operatorname{Rep}^{G} R$ (resp. $V \in I_{G}$ if $G$ is good) we have that $\operatorname{Coker}\left(\xi_{f, V}\right) \otimes \mathcal{O}_{Y, q}$ is defined over $k(q)$, that is $m_{q}\left(\operatorname{Coker}\left(\xi_{f, V}\right) \otimes \mathcal{O}_{Y, q}\right)=0$ where $m_{q}$ denotes the maximal ideal of $\mathcal{O}_{Y, q}$.

In this case $f \in \mathcal{Z}_{G}(Y)$, where $\mathcal{Z}_{G}$ denotes the schematic closure of $\mathrm{B} G$ inside $G$-Cov (see 3.5).

A variant of this result already appeared in my Ph.D. thesis [Ton13b] but under stronger hypotheses on the geometric stabilizers in codimension 1 (see [Ton13b, Theorem 4.4.7]). The proof we present here is different and relies on [Ton15], where a non-equivariant analogue of the above theorem is proved.

We now briefly describe the subdivision of the paper. In the first section we prove Theorem A, while in the second we study the property of induction from an open and closed subgroup. The third section is dedicated to the proof of Theorem B and the fourth section to the proof of Theorem C.

\section{NotATION}

In all the paper we fix a base ring $R$, so that all rings, schemes and stacks will be defined over $R$.

Consider a scheme $T$ and a finite, flat and finitely presented group scheme $G$ over $R$. We denote by $\mathrm{B}_{R} G$ (or simply B $G$ ) the stack over $R$ of $G$-torsors, by Loc $T$ (resp. QCoh $T$ ) the category of sheaves of $\mathcal{O}_{T}$-modules that are locally free of finite rank (resp. quasi-coherent), by $\operatorname{Loc}^{G} T$ (resp. QCoh ${ }^{G} T$ ) the category of sheaves of $\mathcal{O}_{T}$ modules that are locally free of finite rank (resp. quasi-coherent) together with an action of $G$, and by $\mathrm{QAlg}^{G} T$ the category of quasicoherent sheaves of algebras $\mathscr{A}$ on $T$ together with an action of $G$. When $T=\operatorname{Spec} A$ we will often replace $T$ by $A$ and write, for instance, $\operatorname{Loc}^{G} A$ instead of $\operatorname{Loc}^{G}(\operatorname{Spec} A)$. 
If $\mathscr{C}, \mathscr{D}$ are $R$-linear monoidal categories with unities $I, J$ and $\Gamma: \mathscr{C} \longrightarrow \mathscr{D}$ is an $R$-linear functor, a monoidal structure on $\Gamma$ consists of a natural transformation $\iota_{V, W}: \Gamma_{V} \otimes \Gamma_{W} \longrightarrow \Gamma_{V \otimes W}$ for $V, W \in \mathscr{C}$ and a morphism $1: J \longrightarrow \Gamma_{I}$ satisfying certain compatibility conditions. A monoidal structure in which those maps are isomorphisms is called strong. We refer to [Ton14, Definition 2.18] for the precise definition.

Given $\mathcal{F} \in \mathrm{QCoh}^{G} T$ we set $\Omega^{\mathcal{F}}=(\mathcal{F} \otimes-)^{G}: \operatorname{Loc}^{G} R \longrightarrow$ QCoh $T$, which is an $R$-linear functor. If $\mathcal{F} \in \mathrm{QAlg}^{G} T$ then $\Omega^{\mathcal{F}}$ has a monoidal structure induced by the multiplication and the unity of $\mathcal{F}$ (see [Ton14, Proposition 2.22 and Section 4]).

A map $f: X \longrightarrow T$ of schemes is called a cover if it is affine and $f_{*} \mathcal{O}_{\mathcal{X}}$ is locally free of finite rank or, alternatively, if it is finite, flat and finitely presented. Affine maps into a scheme $T$ will be often thought of as quasi-coherent sheaves of algebras on $T$, so that covers correspond to locally free sheaves of algebras of finite rank.

A geometric point of a scheme $T$ is a map $\operatorname{Spec} k \longrightarrow T$, where $k$ is an algebraically closed field.

\section{ACKNOWLEDGEMENT}

I would like to thank Angelo Vistoli and Matthieu Romagny for the useful conversations I had with them and all the suggestions they gave me.

\section{Galois COVERS VIA MONOIDAL FUnCTORS}

The aim of this section is to prove Theorem A. We fix a base ring $R$ and a finite, flat and finitely presented group scheme $G$ over $R$.

Taking into account [Ton14, Remark 4.3 and Theorem 4.6] we have the following result.

Theorem 1.1. The functor $\Omega^{*}$ yields an equivalence between $\mathrm{QCoh}^{G} T\left(\mathrm{QAlg}^{G} T\right)$ and the category of R-linear (monoidal) functors $\operatorname{Loc}^{G} R \longrightarrow$ QCoh $T$ which are left exact on short exact sequences.

Definition 1.2. A $G$-cover of an $R$-scheme $T$ is a cover $f: X \longrightarrow T$ together with an action of $G$ on $X$ such that $f$ is invariant and $f_{*} \mathcal{O}_{X}$ and $R[G] \otimes \mathcal{O}_{T}$ are fppf locally isomorphic as $G$-comodules (not as rings).

We denote by $G$-Cov the stack over $R$ of $G$-covers. The stack $G$-Cov has been introduced in [Ton13a], it is algebraic and of finite type over $R$ and contains $\mathrm{B}_{R} G$ as an open substack.

The following remark (see [Jan87, Part 1, 3.4] for a proof) will be often used in the next pages.

Remark 1.3. If $M \in \mathrm{QCoh}^{G} R$ and $\varepsilon: R[G] \longrightarrow R$ is the counit then the evaluation in $\varepsilon$ yields an $R$-linear isomorphism

$$
\operatorname{Hom}^{G}\left(R[G]^{\vee}, M\right) \simeq M
$$

or, equivalently, the composition $(R[G] \otimes M)^{G} \longrightarrow R[G] \otimes M \stackrel{\varepsilon \otimes \mathrm{id}_{M}}{\longrightarrow} M$ is an $R$-linear isomorphism.

Definition 1.4. Given an $R$-scheme $T$ we denote by $\operatorname{LAlg}^{G} T$ the groupoid of locally free sheaves of algebras over $T$ with an action of $G$ and by $\operatorname{LAlg}_{R}^{G}$ the stack over $R$ they form. Given $n \in \mathbb{N}$ we also denote by $\operatorname{LAlg}_{n}^{G} T$ (resp. $\operatorname{LAlg}_{R, n}^{G}$ ) the subcategory of $\operatorname{LAlg}^{G} T$ (resp. substack of $\operatorname{LAlg}_{R}^{G}$ ) of sheaves of rank $n$.

Proposition 1.5. We have that $\operatorname{LAlg}_{R}^{G}=\sqcup_{n \in \mathbb{N}} \operatorname{LAlg}_{R, n}^{G}$ and that $\operatorname{LAlg}_{R, n}^{G}$ is an algebraic stack of finite presentation over $R$ for all $n \in \mathbb{N}$. Moreover the map

$$
G-\mathrm{Cov} \longrightarrow \operatorname{LAlg}_{R}^{G},(f: X \longrightarrow Y) \longmapsto f_{*} \mathcal{O}_{X}
$$

is an open immersion. 
Proof. The first claim follows from the fact that the rank function for a locally free sheaf is locally constant. For the second one, consider the forgetful functor $\mathrm{LAlg}_{R, n}^{G} \longrightarrow \mathrm{B} \mathrm{GL}_{n}$ and call $X$ the fiber product along the universal torsor $\operatorname{Spec} R \longrightarrow \mathrm{B} \mathrm{GL}_{n}$. For simplicity we can assume that $R[G]$ is free as an $R$-module. The stack $X$ is actually a sheaf $X:(\mathrm{Sch} / R)^{\mathrm{op}} \longrightarrow(\mathrm{Sets})$ and it maps a scheme $T$ to the set of all possible ring structures together with an action of $G$ on $\mathcal{O}_{T}^{n}$. Since a ring structure is given by maps $\mathcal{O}_{T}^{n} \otimes \mathcal{O}_{T}^{n} \longrightarrow \mathcal{O}_{T}^{n}$ (the multiplication) and $\mathcal{O}_{T} \longrightarrow \mathcal{O}_{T}^{n}$ (the unity), while a $R[G]$-comodule structure by a map $\mathcal{O}_{T}^{n} \longrightarrow \mathcal{O}_{T}^{n} \otimes R[G]$ (the comodule structure), we can embed $X$ into an affine space $\mathbb{A}^{N}$. The compatibility conditions among the previous maps allow us to conclude that $X$ is the zero locus in $\mathbb{A}^{N}$ of finitely many polynomials, as required.

We now deal with the last claim. Clearly the map in the statement is fully faithful. We have to prove that if $\mathscr{A} \in \operatorname{LAlg}^{G} B$, where $B$ is a ring, then the locus in Spec $B$ where $\mathscr{A}$ is fppf locally the regular representation is open. Concretely, if $\xi: \operatorname{Spec} k \longrightarrow \operatorname{Spec} B$ is a geometric point and $\mathscr{A} \otimes k \in G$ - $\operatorname{Cov}(k)$ we will prove that there exists a flat and finitely presented map Spec $B^{\prime} \longrightarrow$ Spec $B$ through which $\xi$ factors and such that $\mathscr{A} \otimes B^{\prime} \simeq B^{\prime}[G]$. Denote by $p \in \operatorname{Spec} B$ the image of $\xi$. Both the stack $G$-Cov and $\operatorname{LAlg}_{R}^{G}$ are locally of finite type over $R$ and therefore also the map $G$-Cov $\longrightarrow \operatorname{LAlg}_{R}^{G}$ is so, which in particular implies that $\mathscr{A} \otimes \overline{k(p)} \in G-\operatorname{Cov}(\overline{k(p)})$. Thus we can assume $k=\overline{k(p)}$. Since $k$ is algebraically closed we have that $\mathscr{A} \otimes k$ is the regular representation and thus we have a $G$-equivariant isomorphism $\bar{\omega}: k[G]^{\vee} \longrightarrow(\mathscr{A} \otimes k)^{\vee}$. By 1.3 the map $\bar{\omega}$ is completely determined by a $\bar{\phi} \in \mathscr{A}^{\vee} \otimes k$. There exists a finite field extension $L / k(p)$ such that $\bar{\phi}$ comes from some element in $\mathscr{A}^{\vee} \otimes L$ and it is a general fact that we can find an fppf neighborhood $\operatorname{Spec} B^{\prime}$ of $p$ in $\operatorname{Spec} B$ with a point $p^{\prime} \in \operatorname{Spec} B^{\prime}$ over $p$ such that $k\left(p^{\prime}\right)=L$. Up to shrinking Spec $B^{\prime}$ around $p^{\prime}$ we can assume we have $\phi \in \mathscr{A}^{\vee}$ inducing $\bar{\phi}$. The element $\phi$ defines a $G$-equivariant map $\omega: B[G]^{\vee} \longrightarrow \mathscr{A}^{\vee}$ of locally free sheaves on $A$ inducing $\bar{\omega}$. Since $\bar{\omega}$ is an isomorphism it follows that $\omega$ is an isomorphism in a Zariski open neighborhood of $p$ as required.

Proof of Theorem A, first sentence. Let $A$ be an $R$-algebra. By 1.3 we have

$$
\Omega_{V}^{A[G]}=(A[G] \otimes(V \otimes A))^{G} \simeq V \otimes A \text { for } V \in \operatorname{Loc}^{G} R
$$

More precisely $\Omega^{A[G]}$ is isomorphic to the forgetful functor $\left(-\otimes_{R} A\right): \operatorname{Loc}^{G} R \longrightarrow \operatorname{Loc} A$ as monoidal functor. In particular if $\mathscr{A} \in \mathrm{QAlg}^{G} A$ is fppf locally isomorphic to $A[G]$ (without ring structure) then the functor $\Omega^{\mathscr{A}}=(\mathscr{A} \otimes-)^{G}: \operatorname{Loc}^{G} R \longrightarrow$ QCoh $A$ is fppf locally $R$-linearly isomorphic to the forgetful functor $\left(-\otimes_{R} A\right): \operatorname{Loc}^{G} R \longrightarrow \operatorname{Loc} A$ (without monoidal structure).

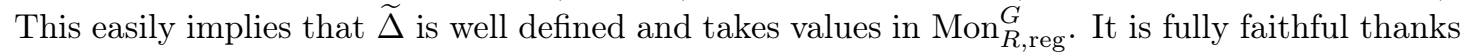
to 1.1. It extends the functor $\Delta$ because if $f: X \longrightarrow \operatorname{Spec} A$ is a $G$-torsor corresponding to $s:$ Spec $A \longrightarrow \mathrm{B}_{R} G$ then $s_{*} \mathcal{O}_{A} \simeq f_{*} \mathcal{O}_{X}$ as sheaves of algebras on $\mathrm{B}_{R} G$ and

$$
\left(s_{*} \mathcal{O}_{A} \otimes_{R} V\right)^{G} \simeq \operatorname{Hom}_{\mathrm{B}_{R} G}\left(V^{\vee}, s_{*} \mathcal{O}_{A}\right) \simeq \operatorname{Hom}_{A}\left(s^{*} V^{\vee}, A\right) \simeq s^{*} V \text { for } V \in \operatorname{Loc}\left(\mathrm{B}_{R} G\right)=\operatorname{Loc}^{G} R
$$

We now prove that it is an open immersion. Let $\Gamma \in \operatorname{Mon}_{R}^{G}(A)$. By 1.1 there exists $\mathscr{A} \in \mathrm{QAlg}^{G} A$ such that $\Gamma \simeq \Omega^{\mathscr{A}}$. By definition of $\operatorname{Mon}_{R}^{G}$ and taking into account 1.3 we also have that $\Omega_{R[G]}^{\mathscr{A}}=(\mathscr{A} \otimes R[G])^{G} \simeq \mathscr{A}$ is a locally free sheaf on $A$, that is $\mathscr{A} \in \operatorname{LAlg}^{G} A$. The result then follows because, by 1.5 , the locus in $\operatorname{Spec} A$ where $\mathscr{A}$ is fppf locally the regular representation is open.

Definition 1.6. The group scheme $G$ is called linearly reductive over $R$ if the functor of invariants

$$
(-)^{G}: \operatorname{Mod}^{G} R \longrightarrow \operatorname{Mod} R
$$

is exact. 
From now until the end of the section we will assume that $G$ is linearly reductive over $R$. Remember that this condition is stable under base change, is local in the fppf topology and that $G$ is fppf locally well-split, which means isomorphic to a semidirect product of a diagonalizable group scheme and a constant group whose order is invertible in the base ring (see [AOV08, Proposition 2.6, Theorem 2.19]). We summarize some properties of linearly reductive groups we are going to use.

Proposition 1.7. Let $T$ be an $R$-scheme and $A$ be an $R$-algebra. Then

1) If $\mathcal{F} \in \mathrm{QCoh}^{G} T$ and $\mathcal{H} \in \mathrm{QCoh} T$ then the natural map

$$
\mathcal{F}^{G} \otimes \mathcal{H} \longrightarrow(\mathcal{F} \otimes \mathcal{H})^{G}
$$

where the action of $G$ on $\mathcal{H}$ is trivial, is an isomorphism. In particular taking invariants $(-)^{G}: \mathrm{QCoh}^{G} T \longrightarrow \mathrm{QCoh} T$ commutes with arbitrary base changes.

2) If $\mathcal{F} \in \mathrm{QCoh}^{G} T$ is locally free of finite rank then the map $\mathcal{F}^{\mathcal{G}} \longrightarrow \mathcal{F}$ locally splits. In particular $\mathcal{F}^{G}$ is locally free of finite rank.

3) Every short exact sequence in $\mathrm{QCoh}^{G} A$ of sheaves in $\operatorname{Loc}^{G} A$ splits. In particular any $R$-linear functor from $\mathrm{Loc}^{G} R$ to an R-linear category is automatically exact.

4) If $R$ is a field any finite-dimensional representation of $G$ is a direct sum of irreducible representations.

Proof. We can assume $T$ affine, say $T=\operatorname{Spec} A$ and replace $\mathcal{F}, \mathcal{H}$ with modules $F, H$ respectively. Point 1) follows because the map in the statement is an isomorphism when $H$ is free and, in general, using a presentation of $H$ and using the exactness of $(-)^{G}$. Point 1 ) implies that $F^{G} \longrightarrow F$ is universally injective, so that point 2) follows from [Mat89, Theorem 7.14] after reducing to a Noetherian base (for instance assuming that $G$ is well-split and, thus, defined over $\mathbb{Z}$ ). For 3 ), if $0 \longrightarrow V \longrightarrow W \longrightarrow Z \longrightarrow 0$ is an exact sequence of sheaves in $\operatorname{Loc}^{G} A$, then $\operatorname{Hom}(W, V) \longrightarrow \operatorname{Hom}(V, V)$ is surjective and, taking invariants, we can find an equivariant splitting. Point 4) follows easily from 3).

We now show an example of a finite, étale and linearly reductive group $G$ over $\mathbb{Q}$ with $\widetilde{\Delta}(G$-Cov $) \neq \operatorname{Mon}_{R, \text { reg }}^{G}($ see Theorem A).

Example 1.8. Consider $R=\mathbb{Q}, G=\mathbb{Z} / 3 \mathbb{Z}, \mathscr{A}=\overline{\mathbb{Q}}[x, y] /(x, y)^{2}$ with the action of $G \times \overline{\mathbb{Q}} \simeq \mu_{3}$ given by $\operatorname{deg} x=\operatorname{deg} y=1$ and $\Gamma=\Omega^{\mathscr{A}}=\left(\mathscr{A} \otimes_{\mathbb{Q}}-\right)^{G}: \operatorname{Loc}^{G} \mathbb{Q} \longrightarrow \operatorname{Loc} \overline{\mathbb{Q}}$. We have that $\mathscr{A} \notin G-\operatorname{Cov}(\overline{\mathbb{Q}})=\mu_{3}-\operatorname{Cov}(\overline{\mathbb{Q}})$ because $\mathscr{A}$ is not isomorphic to the regular representation (it does not contain the $\mu_{3}$-representation corresponding to the character $2 \in \mathbb{Z} / 3 \mathbb{Z}$ ). On the other hand we have $\Gamma \in \operatorname{Mon}_{\mathbb{Q}}^{G}$,reg $(\overline{\mathbb{Q}})$ : the rank condition can be easily checked on the two irreducible representations of $G$ over $\mathbb{Q}$. By 1.1 we can conclude that $\Gamma$ is not in the essential image of the functor $\widetilde{\Delta}: G-\mathrm{Cov} \longrightarrow \operatorname{Mon}_{R}^{G}$.

The problem in the above example is that the group $\mathbb{Z} / 3 \mathbb{Z}$ has a two-dimensional irreducible representation over $\mathbb{Q}$ which splits over $\overline{\mathbb{Q}}$. We want therefore to find a class of linearly reductive groups whose "irreducible" representations are also geometrically irreducible.

Lemma 1.9. Let I be a finite collection of sheaves in $\operatorname{Loc}^{G} R$ which have positive rank in all points of Spec $R$. The following are equivalent:

1) the natural maps

$$
\eta_{M}: \bigoplus_{V \in I} V \otimes_{R} \operatorname{Hom}_{R}^{G}(V, M) \longrightarrow M \text { for } M \in \operatorname{Mod}^{G} R
$$

are isomorphisms. 
2) for all geometric points $\operatorname{Spec} k \stackrel{\xi}{\longrightarrow} \operatorname{Spec} R$ the set $\left\{V \otimes_{R} k\right\}_{V \in I}$ is a set of representatives of the irreducible representations of $G \times k$ and $V \otimes_{R} k \simeq W \otimes_{R} k$ if and only if $V=W$.

3) (assuming $\operatorname{Spec} R$ connected) there exists a geometric point $\operatorname{Spec} k \stackrel{\xi}{\longrightarrow} \operatorname{Spec} R$ for which the set $\left\{V \otimes_{R} k\right\}_{V \in I}$ is a set of representatives of the irreducible representations of $G \times k$ and $V \otimes_{R} k \simeq W \otimes_{R} k$ if and only if $V=W$.

In the above cases we have that $\operatorname{Hom}^{G}(V, W)=0$ if $V \neq W \in I$ and $\operatorname{Hom}^{G}(V, V)=R_{V}$ if $V \in I$.

Proof. We are going to use that taking invariants commutes with arbitrary base changes (see 1.7). If Spec $k \longrightarrow \operatorname{Spec} R$ is a geometric point we set $G_{k}=G \times k$.

1) $\Longrightarrow 2$ ). If Spec $k \longrightarrow \operatorname{Spec} R$ is a geometric point and $M \in \operatorname{Mod}^{G_{k}} k$ then $\operatorname{Hom}_{R}^{G}(V, M) \simeq$ $\operatorname{Hom}_{k}^{G_{k}}(V \otimes k, M)$ and $\eta_{M} \simeq\left(\eta_{M}\right) \otimes k$. Thus we can assume that $R$ is an algebraically closed field. In this case the result follows by decomposing representations into irreducible ones.

$2), 3) \Longrightarrow 1$ ). If $V, W \in \operatorname{Loc}^{G} R$ then $\operatorname{Hom}^{G}(V, W)$ is locally free by $\left.1.7,2\right)$. Thus, checking the rank on the geometric points (on the given geometric point if Spec $R$ is connected), if $V, W \in I$ then $\operatorname{Hom}^{G}(V, W)=0$ for $V \neq W$ and $\operatorname{Hom}^{G}(V, V)=R \operatorname{Rid}_{V}$. In particular if $\operatorname{Spec} k \stackrel{\xi}{\longrightarrow} \operatorname{Spec} R$ is any geometric point then $\xi^{*}: I_{G} \longrightarrow \operatorname{Loc}^{G} k$ is injective onto a subset of representatives of the irreducible representations of $G \times k$. Given $M \in \operatorname{Mod}^{G} R$ we therefore have that $\xi^{*} \eta_{M}$ is injective and, if $\xi^{*}\left(I_{G}\right)$ is a full set of representatives of irreducible representations of $G \times k$, an isomorphism. If Spec $R$ is connected, so that $R[G]$ has constant rank, applying this consideration to $M=R[G]$ and using 1.3 we can conclude that 3$) \Longrightarrow 2$ ) by dimension. In particular $\eta_{M}$ is an isomorphism on all geometric points of $\operatorname{Spec} R$. If $M$ is an arbitrary direct sum of locally free $G$-comodules of finite rank it follows that $\eta_{M}$ is an isomorphism. In general, using 1.3, we can find an exact sequence of $G$-comodules $V_{1} \longrightarrow V_{0} \longrightarrow M \longrightarrow 0$ where the $V_{i}$ are sum of copies of $R[G]^{\vee}$. Since $\eta_{V_{0}}, \eta_{V_{1}}$ are isomorphisms, by functoriality it follows that $\eta_{M}$ is an isomorphism as well.

Remark 1.10. If $I$ is a collection of sheaves satisfying the conditions in 1.9, then there exists another collection $I^{\prime}$ satisfying the same conditions and such that $R \in I$. Indeed notice first that, if $R=R_{1} \times R_{2}$ and we are able to replace the collections $I_{\mid \operatorname{Spec} R_{1}}$ and $I_{\mid \text {Spec } R_{2}}$ then we can easily replace the collection $I$. In particular, since the map $\eta_{R}$ in 1.9 is an isomorphism, we can assume there exists $V \in I$ such that $V \otimes \operatorname{Hom}^{G}(V, R) \longrightarrow R$ is an isomorphism, which means that $V$ is an invertible sheaf with the trivial action of $G$. If we replace $V$ by $R$ in $I$ we find the desired collection.

Definition 1.11. We will say that $G$ has a good representation theory over $R$ if it admits a collection $I$ as in 1.9. A good linearly reductive group is a pair $\left(G, I_{G}\right)$ where $G$ is a finite, flat, finitely presented and linearly reductive group scheme over $R$ and $I_{G}$ is a collection as in 1.9. We will simply write $G$ if this will not lead to confusion. For simplicity we will also assume that $R \in I_{G}$ (see 1.10).

If $R \longrightarrow R^{\prime}$ is a morphism and $G$ is a good linearly reductive group, then $G \times R^{\prime}$ is naturally a good linearly reductive group with the collection of the pullbacks of the modules in $I_{G}$.

Remark 1.12. All diagonalizable group schemes are good over the integers, while if $R$ is a field, then $G$ is good if and only if its irreducible representations are geometrically irreducible.

We are going to prove that any linearly reductive group is fppf locally good.

Lemma 1.13. Let $\mathcal{X}$ be a proper and flat algebraic stack over a Noetherian local ring $R$. Denote by $k$ the residue field of $R$ and consider a locally free sheaf $V_{0}$ of rank $n$ over $\mathcal{X} \times k$. If $\mathrm{H}^{2}(\mathcal{X} \times$ 
$\left.k, \underline{\text { End }}\left(V_{0}\right)\right)=0$, then there exists a locally free sheaf of rank $n$ over $\mathcal{X} \times \widehat{R}$ lifting $V_{0}$, where $\widehat{R}$ is the completion of $R$.

Proof. Taking into account Grothendieck's existence theorem for proper stacks, we can assume that $R$ is an Artinian ring (so that $\widehat{R} \simeq R$ ) and that we have a lifting $\bar{V}$ of $V_{0}$ over $\mathcal{X} \times(R / I)$, where $I$ an ideal of $R$ such that $I^{2}=0$. Define the stack $\mathcal{Y}$ over the small fppf site $\mathcal{X}_{\text {fppf }}$ of $\mathcal{X}$ whose objects over $\operatorname{Spec} B \longrightarrow \mathcal{X}$ are locally free sheaves $N$ of rank $n$ over $B$ with an isomorphism $\phi: N \otimes(B / I B) \longrightarrow \bar{V} \otimes(B / I B)$. A section of $\mathcal{Y} \longrightarrow \mathcal{X}_{\text {fppf }}$ yields a lifting of $\bar{V}$ on $\mathcal{X}$. We are going to prove that $\mathcal{Y}$ is a gerbe over $\mathcal{X}_{\text {fppf }}$ banded by the sheaf of abelian groups $\pi_{*}$ End $\left(V_{0}\right)$, where $\pi: \mathcal{X} \times k \longrightarrow \mathcal{X}$ is the obvious closed immersion. Since $\mathrm{H}^{2}\left(\mathcal{X}, \pi_{*} \operatorname{End}\left(V_{0}\right)\right)=$ $\mathrm{H}^{2}\left(\mathcal{X} \times k\right.$, End $\left.\left(V_{0}\right)\right)=0$ parametrizes those gerbes (see [Gir71, Chapter IV, §3, Section 3.4]), we can then conclude that $\mathcal{Y} \longrightarrow \mathcal{X}_{\text {fppf }}$ is a trivial gerbe, which means that it has a section as required.

I claim that $\bar{V}$ is trivial in the fppf topology of $\mathcal{X}$, which implies that $\mathcal{Y} \longrightarrow \mathcal{X}_{\text {fppf }}$ has local sections. Indeed if $B$ is a ring and $P \longrightarrow \operatorname{Spec} B / I B$ is a $\mathrm{Gl}_{n}$-torsor then by standard deformation theory it extends to a smooth map $Q \longrightarrow \operatorname{Spec} B$. In particular, if we base change to $Q$, we can conclude that $P$ over $Q \times(B / I B)$ has a section, which means that it is trivial.

I also claim that two objects of $\mathcal{Y}$ over the same object of $\mathcal{X}_{\text {fppf }}$ are locally isomorphic. Replacing again locally free sheaves by $\mathrm{Gl}_{n}$-torsors, given $\mathrm{Gl}_{n}$-torsors $P, Q$ over $\operatorname{Spec} B$, we have to show that an equivariant isomorphism $P \times(B / I B) \longrightarrow Q \times(B / I B)$ locally extends to an equivariant isomorphism $P \longrightarrow Q$. In particular we can assume that $P$ and $Q$ are both trivial and in this case the above property follows because $\mathrm{Gl}_{n}(B) \longrightarrow \mathrm{Gl}_{n}(B / I B)$ is surjective, since $\mathrm{Gl}_{n}$ is smooth.

The previous two claims show that $\mathcal{Y} \longrightarrow \mathcal{X}_{\text {fppf }}$ is a gerbe. We have now to check the banding and therefore to compute the automorphism group of an object $(N, \phi) \in \mathcal{Y}$ over a ring $B$. The group $\operatorname{Aut}(\chi)$ consists of the automorphism $N \stackrel{\lambda}{\longrightarrow} N$ inducing the identity on $N / I N$. It is easy to check that the map

$$
\operatorname{Hom}_{B}(N, I N) \longrightarrow \text { Aut } \chi, \delta \longmapsto \operatorname{id}_{N}+\delta
$$

is an isomorphism of groups. Since $I N=I \otimes_{R} N$ and $N \otimes\left(B / m_{R} B\right) \simeq V_{0} \otimes\left(B / m_{R} B\right)$ we have

$$
\operatorname{Hom}_{B}(N, I N)=I \otimes \operatorname{End}_{B}(N) \simeq I / I^{2} \otimes \operatorname{End}_{B}(N) \simeq \operatorname{End}_{B / m_{R} B}\left(V_{0} \otimes\left(B / m_{R} B\right)\right)
$$

Lemma 1.14. Assume that $R$ is a Henselian ring with residue field $k$. The any finite dimensional representation of $G$ over $k$ lifts to $R$.

Proof. Since $G$ is finitely presented, we can assume that $R$ is the Henselization of a scheme of finite type over $\mathbb{Z}$. Since $G$ is linearly reductive, we have that $\mathrm{H}^{2}(\mathrm{~B}(G \times k),-)=0$ and, viewing $G$-representations as sheaves over B $G$ and using 1.13, we obtain a lifting of $V$ to a representation over the completion $\widehat{R}$. We can then conclude using Artin's approximation theorem over $R$.

Proposition 1.15. There exists an fppf covering $\mathcal{U}=\left\{U_{i} \longrightarrow \operatorname{Spec} R\right\}_{i \in I}$ such that $G \times_{S} U_{i}$ has a good representation theory over $U_{i}$ for all $i$. If $G$ is étale over $R$ there exists an étale covering with the same property.

Proof. We start with the case when $R=k$ is a field. The group $G$ is good after a finite extension of $k$ because an irreducible representation of $G$ over the algebraic closure of $k$ is always defined over a finite extension of $k$. Now assume that $G$ is étale. If $k$ is perfect there is nothing to prove. So assume char $k=p>0$. After passing to a separable extension of $k$ we can assume that $G$ is constant of order prime to $p$. So $G$ is defined over $\mathbb{F}_{p}$, which is perfect and again we have our claim. 
Now return to the general case. Since $G$ is finitely presented, we can assume that $R$ is of finite type over $\mathbb{Z}$. Let $p \in \operatorname{Spec} R$ and $L / k(p)$ an extension such that $G_{L}=G \times L$ is good, with $L / k(p)$ separable if $G$ is étale. There exists a flat finitely presented map $h: \operatorname{Spec} R^{\prime} \longrightarrow \operatorname{Spec} R$ such that $h^{-1}(p) \simeq \operatorname{Spec} L$. If $L / k$ is separable we can even assume that $h$ is étale. This shows that we can assume that $G_{k(p)}=G \times k(p)$ is good. From 1.14 any $G_{k(p)}$ representation lifts to $R_{p}^{h}$, the Henselization of $R_{p}$, and, since this ring is a direct limit of algebras étale over $R$, we get the required result.

Putting together 1.14 and 1.15 we get:

Theorem 1.16. A constant linearly reductive group over a strictly Henselian ring has a good representation theory.

Remark 1.17. If $\left(G, I_{G}\right)$ is a good linearly reductive group there is an explicit way to map linear functors to sheaves, which may be useful in concrete examples. Let $T$ be an $R$-scheme, $\operatorname{set}_{R}^{G}(T)$ for the category of $R$-linear functors $\operatorname{Loc}^{G} R \longrightarrow$ QCoh $T$ and define

$$
\mathcal{F}_{*}: \mathrm{L}_{R}^{G}(T) \longrightarrow \mathrm{QCoh}^{G} T, \mathcal{F}_{\Gamma}=\bigoplus_{V \in I_{G}} V^{\vee} \otimes \Gamma_{V}
$$

where the action of $G$ on the $\Gamma_{V}$ is trivial. Using 1.9 it is easy to see that $\mathcal{F}_{*}$ is a quasi-inverse of $\Omega^{*}: \mathrm{QCoh}^{G} T \longrightarrow \mathrm{L}_{R}^{G}(T), \Omega^{\mathcal{G}}=(\mathcal{G} \otimes-)^{G}$, the other natural isomorphism being

$$
\beta_{U}: \Omega_{U}^{\mathcal{F}_{\Gamma}} \simeq\left(U \otimes \mathcal{F}_{\Gamma}\right)^{G} \simeq \bigoplus_{V \in I_{G}} \operatorname{Hom}^{G}(V, U) \otimes \Gamma_{V} \longrightarrow \Gamma_{U} \text { for } \Gamma \in \mathrm{L}_{R}^{G}(T), U \in \operatorname{Loc}^{G} R
$$

The map $\beta_{U}^{-1}: \Gamma_{U} \longrightarrow\left(U \otimes \mathcal{F}_{\Gamma}\right)^{G}$ is uniquely determined by a map $\alpha_{U}: U^{\vee} \otimes \Gamma_{U} \longrightarrow \mathcal{F}_{\Gamma}$. It is easy to see that:

1) if $U \in I_{G}$ then $\alpha_{U}$ is the inclusion;

$2)$ if $U=U_{1} \oplus U_{2}$ then $\alpha_{U}$ is zero on $U_{i}^{\vee} \otimes \Gamma_{U_{j}}$ for $i \neq j \in\{1,2\}$ and coincides with $\alpha_{U_{i}}$ on $U_{i}^{\vee} \otimes \Gamma_{U_{i}}$ for all $i=1,2$;

3) if $U=\mathcal{H} \otimes U^{\prime}$ for $\mathcal{H} \in \operatorname{Loc} R$ and $U^{\prime} \in \operatorname{Loc}^{G} R$ then $\alpha_{U}$ is

$$
U^{\vee} \otimes \Gamma_{U} \simeq \mathcal{H}^{\vee} \otimes \mathcal{H} \otimes U^{\prime} \otimes \Gamma_{U^{\prime}} \stackrel{\mathrm{ev} \mathcal{H} \otimes \alpha_{U^{\prime}}}{\longrightarrow} \mathcal{F}_{\Gamma}
$$

where $\mathrm{ev}_{\mathcal{H}}: \mathcal{H}^{\vee} \otimes \mathcal{H} \longrightarrow R$ is the evaluation;

4) if $\gamma: V \longrightarrow U$ is a $G$-equivariant isomorphism then $\alpha_{V}=\alpha_{U} \circ\left[\left(\gamma^{\vee}\right)^{-1} \otimes \Gamma_{\gamma}\right]$.

Using the maps $\alpha_{*}$ (and by going through the definitions) if $\Gamma$ is a monoidal functor the associated ring structure on $\mathcal{F}_{\Gamma}$ is given by

$$
V^{\vee} \otimes \Gamma_{V} \otimes W^{\vee} \otimes \Gamma_{W} \longrightarrow(V \otimes W)^{\vee} \otimes \Gamma_{V \otimes W} \stackrel{\alpha_{V \otimes W}}{\longrightarrow} \mathcal{F}_{\Gamma} \text { for } V, W \in I_{G}
$$

Proof of Theorem A, last sentence. The functor $\widetilde{\Delta}: \operatorname{LAlg}_{R}^{G} \longrightarrow \operatorname{Mon}_{R}^{G}$ is well defined thanks to 1.7. It is an equivalence thanks to 1.1 and the fact that if $\mathscr{A} \in \mathrm{QAlg}^{G} T$ and $\Omega^{\mathscr{A}} \in \operatorname{Mon}_{R}^{G}(T)$ then, using $1.3, \mathscr{A} \simeq(\mathscr{A} \otimes R[G])^{G}=\Omega_{R[G]}^{\mathscr{A}}$ is locally free of finite rank.

We now show the last equality in the statement. Using notation from 1.17, if $\Gamma \in \operatorname{Mon}_{R, \mathrm{reg}}^{G}(T)$ then $\mathscr{A}=\mathcal{F}_{\Gamma} \in \mathrm{QAlg}^{G} T$ is such that $\Gamma \simeq \Omega^{\mathscr{A}}$. We can assume that $\Gamma_{V}$ is free of rank rk $V$ for all $V \in I_{G}$. In this case $R[G] \otimes \mathcal{O}_{T}$ and $\mathscr{A}$ have the same decomposition in terms of the representations in $I_{G}$ and thus they are isomorphic.

We finally show that $G$-Cov is open and closed in $\operatorname{LAlg}_{R}^{G}$. This problem is fppf local in the base, thus we can assume that $G$ is a good linearly reductive group thanks to 1.15 . In this case $G$-Cov (resp. $\operatorname{LAlg}_{R}^{G}$ ) corresponds to $\operatorname{Mon}_{R \text {,reg }}^{G}\left(\right.$ resp. $\operatorname{Mon}_{R}^{G}$ ) via $\widetilde{\Delta}$ and $\operatorname{Mon}_{R \text {,reg }}^{G}$ is the locus in $\operatorname{Mon}_{R}^{G}$ of functors $\Gamma$ such that $\operatorname{rk} \Gamma_{V}=\operatorname{rk} V$ for all $V \in I_{G}$. Since $I_{G}$ is finite, this is an open and closed condition, as required. 


\section{INDUCTION FROM A SUBGROUP FOR EQUIVARIANT ALGEBRAS.}

As in the previous section we fix a base ring $R$ and a flat, finite and finitely presented group scheme $G$ over $R$.

Let $H$ be an open and closed subgroup scheme of $G$. If $\mathcal{F} \in \mathrm{QCoh}^{H} T$ we define the induction from $H$ to $G$ of $\mathcal{F}$, denoted by $\operatorname{ind}_{H}^{G} \mathcal{F}$, as $(\mathcal{F} \otimes R[G])^{H} \in \mathrm{QCoh}^{G} T$. For details and properties we refer to [Jan87, Part I, Section 3]. If $\mathcal{F}$ is also a quasi-coherent sheaf of algebras, that is $\mathcal{F} \in \mathrm{QAlg}^{H} T$, then $\operatorname{ind}_{H}^{G} \mathcal{F} \in \mathrm{QAlg}^{G} T$, that is it inherits a natural structure of sheaf of algebras with an action of $G$. The aim of this section is to prove the following.

Theorem 2.1. If $H$ is an open and closed subgroup scheme of $G$ the functor

$$
\operatorname{ind}_{H}^{G}: \operatorname{LAlg}_{R}^{H} \longrightarrow \operatorname{LAlg}_{R}^{G}, \mathscr{A} \longmapsto(\mathscr{A} \otimes R[G])^{H}
$$

is well defined, quasi-affine and étale. The (open) image consists of those $\mathscr{A} \in \operatorname{LAlg}_{R}^{G} T$ such that, for all geometric points $\operatorname{Spec} k \longrightarrow T$, there exists a subset of points of $\operatorname{Spec}(\mathscr{A} \otimes k)$ whose geometric stabilizers are contained in $H \times k$ and whose $G(k)$-orbits cover the whole $\operatorname{Spec}(\mathscr{A} \otimes k)$.

Lemma 2.2. Assume that $R$ is a strictly Henselian ring. If $A, B$ are local $R$-algebras such that $A$ is finite over $R$ and the maximal ideal of $B$ lies over the maximal ideal of $R$, then $A \otimes_{R} B$ is local.

Proof. Set $k_{A}, k_{B}$ for their residue fields. Since $A \otimes_{R} B$ is finite over $B$ it is enough to note that $k_{A} \otimes_{k_{R}} k_{B}$ is local since $k_{A} / k_{R}$ is purely inseparable.

Lemma 2.3. Assume that $R$ is a strictly Henselian ring and let $X \longrightarrow \operatorname{Spec} R$ be a cover with an action of $G$. Consider the decomposition into connected components

$$
G=\bigsqcup_{i \in \underline{G}} G_{i} \text { and } X=\bigsqcup_{j \in \underline{X}} X_{j}
$$

Given $i \in \underline{G}$ and $j \in \underline{X}$ the restriction of the action $X_{j} \times G_{i} \longrightarrow X$ factors through a unique component $X_{j \star i}$ with $j \star i \in \underline{X}$. The operation $-\star-: \underline{G} \times \underline{G} \longrightarrow \underline{G}$ obtained when $X=G$ with the right action of $G$ by multiplication makes $\underline{G}$ into a group, whose unity $1 \in \underline{G}$ is the connected component containing the identity. In general the association $\underline{X} \times \underline{G} \longrightarrow \underline{X}$ defines a right action of $\underline{G}$ on the set $\underline{X}$. Moreover $G_{1}$ is a subgroup scheme of $G$ and the map $G_{i} \times G_{1} \longrightarrow G_{i}$ makes $G_{i}$ into a $G_{1}$-torsor for all $i \in \underline{G}$.

Proof. Finite algebras over Henselian rings are products of their localizations. In particular the $G_{i}$ and $X_{j}$ are the spectrum of the localizations of $\mathrm{H}^{0}\left(\mathcal{O}_{G}\right)$ and $\mathrm{H}^{0}\left(\mathcal{O}_{X}\right)$ respectively. All the conclusions follow easily from 2.2.

Lemma 2.4. Let $H$ be an open and closed subgroup scheme of $G$ and let $B$ be a local ring with residue field $k, \mathscr{A} \in \operatorname{LAlg}^{G} B, Z=\operatorname{Spec} \widetilde{\mathscr{A}} \subseteq \operatorname{Spec} \mathscr{A}$ be an $H$-equivariant open and closed subscheme. Then the map $\mathscr{A} \longrightarrow \operatorname{ind}_{H}^{G} \widetilde{\mathscr{A}}$ induced by the projection $\mathscr{A} \longrightarrow \widetilde{\mathscr{A}}$ is an isomorphism if and only if

$$
(Z \times \bar{k}) g \cap Z \times \bar{k} \neq \emptyset \Longrightarrow g \in H(\bar{k}) \quad \forall g \in G(\bar{k})
$$

and the $G(\bar{k})$-orbits of $Z \times \bar{k}$ cover the whole $\operatorname{Spec}(\mathscr{A} \otimes \bar{k})$. In this case $\widetilde{\mathscr{A}} \in \mathrm{LAlg}^{H} B$ and the geometric stabilizers of $Z$ for the action of $H$ or $G$ coincide. If in addition $G$ is étale over $B$, then we can replace $\bar{k}$ with the separable closure of $k$ in the formula above.

Proof. It is easy to see that there exists a (étale if $G / R$ is étale) cover Spec $R^{\prime} \longrightarrow \operatorname{Spec} R$ such that $G \times R^{\prime}$ splits as disjoint union of copies of $H \times R^{\prime}$, that is the right cosets of $H \times R^{\prime}$. Localizing in a maximal ideal of $R^{\prime}$ we see that we can assume this decomposition holds also for 
$R$ and that $R=B$. In particular $R[G] \simeq R[H]^{\mathcal{R}}$, where $\mathcal{R} \subseteq G(R)$ is a set of representatives of the right cosets of $H$, and therefore, using 1.3 , we have

$$
\operatorname{ind}_{H}^{G} \widetilde{\mathscr{A}}=(\widetilde{\mathscr{A}} \otimes R[G])^{H} \simeq\left(\widetilde{\mathscr{A}} \otimes R[H]^{\mathcal{R}}\right)^{H} \simeq\left((\widetilde{\mathscr{A}} \otimes R[H])^{H}\right)^{\mathcal{R}} \simeq \widetilde{\mathscr{A}}^{\mathcal{R}}
$$

In particular $\operatorname{ind}_{H}^{G} \widetilde{\mathscr{A}}$ is flat over $B$ and, if $\mathscr{A} \simeq \operatorname{ind}_{H}^{G} \widetilde{\mathscr{A}}$, then $\widetilde{\mathscr{A}}$ is locally free and therefore $\widetilde{\mathscr{A}} \in \mathrm{LAlg}^{H} B$. Since the map $\mathscr{A} \longrightarrow \operatorname{ind}_{H}^{G} \widetilde{\mathscr{A}}$ is an isomorphism if and only if it is so after tensoring with $\bar{k}$ or the separable closure $k^{s}$, we can assume that $R=B=L$ is $k^{s}$ if $G / B$ is étale or $\bar{k}$ otherwise. The action of $G$ on $\operatorname{ind}_{H}^{G} \widetilde{\mathscr{A}} \simeq \widetilde{\mathscr{A}^{\mathcal{R}}}$ is induced by the right action of $G(L)$ on $\mathcal{R}$ and the the action of $H$ on $\widetilde{\mathscr{A}}$. Thus the map

$$
\operatorname{Spec}\left(\operatorname{ind}_{H}^{G} \widetilde{\mathscr{A}}\right)=\bigsqcup_{g \in \mathcal{R}} Z \longrightarrow \operatorname{Spec} \mathscr{A}
$$

is the disjoint union of the $g_{\mid Z}: Z \longrightarrow$ Spec $\mathscr{A}$ where $g_{\mid Z}$ is the restriction of the action of $g \in G(L)$. Taking into account 2.3, the above map is an isomorphism if and only if Spec $\mathscr{A}$ is the disjoint union of the $Z g$ for $g \in \mathcal{R}$, which is equivalent to the two conditions given in the statement.

Definition 2.5. If $R$ is a strictly Henselian ring, $X \longrightarrow \operatorname{Spec} R$ a cover with an action of $G$ and $X_{i}$ a connected component of $X$ we call the stabilizer of $X_{i}$ the open and closed subgroup $H$ of $G$ which is the disjoint union of the components $G_{j}$ of $G$ such that $X_{i} G_{j} \subseteq X_{i}$.

Lemma 2.6. Assume that $R$ is a strictly Henselian ring with residue field $k$ and let $\mathscr{A} \in$ $\operatorname{LAlg}^{G} R, p \in \operatorname{Spec} \mathscr{A}$ be a maximal ideal and denote by $H_{p}$ the geometric stabilizer of $p$ and by $U_{p}$ the stabilizer of the connected component $\mathrm{Spec} \mathscr{A}_{p}$. Then $H_{p}$ is a closed subgroup scheme of $U_{p} \times \bar{k}$, they are topologically equal and, if $G(\bar{k})$ acts transitively on $\operatorname{Spec}(\mathscr{A} \otimes \bar{k})$, there exists an isomorphism

$$
\operatorname{ind}_{U_{p}}^{G} \mathscr{A}_{p} \simeq \mathscr{A}
$$

Proof. We are going to use 2.2 several times. Set $X=\operatorname{Spec} \mathscr{A}$ and $X_{p}=$ Spec $\mathscr{A}_{p}$. Notice that the closed points of $\operatorname{Spec} \mathscr{A}$ correspond to $\operatorname{Spec}(\mathscr{A} \otimes k)$ or $\operatorname{Spec}(\mathscr{A} \otimes \bar{k})$, so that we can also think $p \in \operatorname{Spec}(\mathscr{A} \otimes \bar{k})$. Moreover $U_{p} \times \bar{k}$ is the stabilizer of the connected component Spec $\mathscr{A}_{p} \otimes \bar{k}$ of Spec $\mathscr{A} \otimes \bar{k}$. In particular $H_{p}(\bar{k})=U_{p}(\bar{k})$ so that $H_{p}$ is a closed subgroup scheme of $G \times \bar{k}$ contained in $U_{p} \times \bar{k}$. Moreover we can apply 2.4 with $Z=\operatorname{Spec} \mathscr{A}_{p}$ and $H=U_{p}$ obtaining the desired isomorphism.

Proof of Theorem 2.1. Arguing as in the proof of 2.4, we can assume that $G$ is a disjoint union of copies of $H$, namely its right cosets, obtaining an isomorphism

$$
\operatorname{ind}_{H}^{G} \mathscr{B}=(\mathscr{B} \otimes R[G])^{H} \simeq\left((\mathscr{B} \otimes R[H])^{H}\right)^{\mathcal{R}} \simeq \mathscr{B}^{\mathcal{R}} \text { for } \mathscr{B} \in \operatorname{LAlg}_{R}^{H}
$$

where $\mathcal{R} \subseteq G(R)$ is a set of representatives of the right cosets of $H$ in $G$. This shows that $\operatorname{ind}_{H}^{G}$ is well defined. Moreover, since it is faithful, it is also representable by algebraic spaces. We are going to prove that it is étale and separated. By [MBL99, Appendice A, Theorem A.2] it will follow that it is quasi-affine.

Let $A$ be an $R$-algebra and $\xi: \operatorname{Spec} A \longrightarrow \operatorname{LAlg}_{R}^{G}$ be a map given by $\mathscr{A} \in \operatorname{LAlg}^{G} A$. The fiber product $X:(\operatorname{Sch} / A)^{\mathrm{op}} \longrightarrow(\mathrm{Sets})$ of $\xi$ and $\operatorname{ind}_{H}^{G}$ is given by

$$
X(T)=\left\{(\mathscr{B}, \psi) \mid \mathscr{B} \in \operatorname{LAlg}^{H} T \text { and } \psi: \mathscr{A} \otimes \mathcal{O}_{T} \simeq \operatorname{ind}_{H}^{G} \mathscr{B}\right\}
$$

Notice that the datum $\psi$ can also be given as an $H$-equivariant map $\mathscr{A} \otimes \mathcal{O}_{T} \longrightarrow \mathscr{B}$ which induces an isomorphism $\mathscr{A} \otimes \mathcal{O}_{T} \longrightarrow \operatorname{ind}_{H}^{G} \mathscr{B}$ via adjunction. In particular we obtain a map $X \longrightarrow \operatorname{Hilb}_{\mathrm{Spec} \mathscr{A} / A}$ which is a monomorphism because if $(\mathscr{B}, \psi) \in X$ then the action of $H$ 
on $\mathscr{B}$ is completely determined by the action of $H$ on $\mathscr{A}$ and by $\psi$. Since $\mathrm{Hilb}_{\mathrm{Spec} \mathscr{A} / R}$ and monomorphisms are separated, it follows that $X$ is separated too.

Since $\operatorname{LAlg}_{R}^{H}$ and $\operatorname{LAlg}_{R}^{G}$ are locally of finite presentation by 1.5 so is $X \longrightarrow \operatorname{Spec} A$. Thus in order to show that $X$ is étale over $A$ we can assume that $A$ is an Artinian local ring and prove that, if $J$ is a square zero ideal of $A$, then an object $\left(\mathscr{B}^{\prime}, \psi^{\prime}\right) \in X(A / J)$ extends uniquely to $X(A)$. The map Spec $\mathscr{B}^{\prime} \longrightarrow \operatorname{Spec} \mathscr{A} / J \mathscr{A}$ induced by $\psi^{\prime}$ is an $H$-invariant open and closed subscheme of $\operatorname{Spec} \mathscr{A} / J \mathscr{A}$. This gives an open and closed subscheme $\operatorname{Spec} \mathscr{B} \subseteq$ Spec $\mathscr{A}$. This is also $H$ invariant: if $\gamma: \operatorname{Spec} \mathscr{B} \times H \longrightarrow \operatorname{Spec} \mathscr{A}$ is the restriction of the action, then $\gamma^{-1}$ (Spec $\mathscr{A}-$ Spec $\mathscr{B})=\emptyset$ because it is empty after tensoring by $A / J$. Thus we have extended the $H$ equivariant map

$$
\mathscr{A} \otimes A / J \stackrel{\psi}{\longrightarrow} \operatorname{ind}_{H}^{G} \mathscr{B}^{\prime} \longrightarrow \mathscr{B}^{\prime}
$$

to an $H$-equivariant map $\mathscr{A} \longrightarrow \mathscr{B}$ and it is also clear that this extension is unique up to a unique isomorphism. Finally the map $\mathscr{A} \longrightarrow \operatorname{ind}_{H}^{G} \mathscr{B}$ is an isomorphism because it is so after tensoring by $A / J$.

It remains to characterize the image of $\operatorname{ind}_{H}^{G}$. Let $k$ be an algebraically closed field and $\mathscr{A} \in \operatorname{LAlg}^{G} k$. Given $p \in \operatorname{Spec} \mathscr{A}$ we denote by $H_{p}$ its geometric stabilizer and by $U_{p}$ the stabilizer of Spec $\mathscr{A}_{p}$.

Assume that $\mathscr{A}$ is in the image, that is $\mathscr{A} \simeq \operatorname{ind}_{H}^{G} \mathscr{B}$. The conclusion follows applying 2.4 with $\widetilde{\mathscr{A}}=\mathscr{B}$. Conversely assume there is a set of points $Z \subseteq$ Spec $\mathscr{A}$ as in the statement. Set $X=\operatorname{Spec} \mathscr{A}$ and $X_{p}=\operatorname{Spec} \mathscr{A}_{p}$ for $p \in \operatorname{Spec} \mathscr{A}$. We can assume that the points of $Z$ are all in different orbits, that is

$$
X=\bigsqcup_{p \in Z} X_{p} G(k)
$$

By 2.4 we have $U_{p}(k)=H_{p}(k)$ and therefore $U_{p} \subseteq H$. Moroever we also have

$$
\mathscr{A} \simeq \prod_{p \in Z} \operatorname{ind}_{U_{p}}^{G} \mathscr{A}_{p} \simeq \prod_{p \in Z} \operatorname{ind}_{H}^{G}\left(\operatorname{ind}_{U_{p}}^{H} \mathscr{A}_{p}\right) \simeq \operatorname{ind}_{H}^{G}\left(\prod_{p \in Z} \operatorname{ind}_{U_{p}}^{H} \mathscr{A}_{p}\right)
$$

as required.

We conclude with the following results that will be used in the next sections.

Corollary 2.7. Assume that $G$ is a constant group and let $\mathscr{A} \in \operatorname{LAlg}^{G} B$, where $B$ is an $R$ algebra, such that $\mathscr{A}^{G}=B$. If $H$ is the geometric stabilizer of a prime ideal $p$ of $\mathscr{A}$ lying over $q \in \operatorname{Spec} B$ then there exists a an étale morphism $B \longrightarrow B^{\prime}, q^{\prime} \in \operatorname{Spec} B^{\prime}$ over $q, \widetilde{A} \in \operatorname{LAlg}^{H} B^{\prime}$ such that $\widetilde{\mathscr{A}^{H}}=B^{\prime}$ and a $G$-equivariant isomorphism

$$
\mathscr{A} \otimes_{B} B^{\prime} \simeq \operatorname{ind}_{H}^{G} \widetilde{\mathscr{A}}
$$

Moreover we can also assume that $\widetilde{\mathscr{A}} \otimes \overline{k\left(q^{\prime}\right)}$ is local, its maximal ideal lies over $p \in \operatorname{Spec} \mathscr{A}$ and has geometric stabilizer equal to $H$.

Proof. We are going to prove that $G(\overline{k(q)})$ acts transitively on $\operatorname{Spec}(\mathscr{A} \otimes \overline{k(q)})$. Using 2.2 , we can find a separable finite extension $L / k$ such that $\operatorname{Spec}(\mathscr{A} \otimes \overline{k(q)}) \longrightarrow \operatorname{Spec}(\mathscr{A} \otimes L)$ is bijective. Moreover there exists a flat and local $B$-algebra $B^{\prime}$ with residue field $L$. Since $\left(\mathscr{A} \otimes B^{\prime}\right)^{G}=B^{\prime}$, by standard arguments it follows that $G$ (as constant group) acts transitively on the set of maximal ideals of $\mathscr{A} \otimes B^{\prime}$ and thus on $\operatorname{Spec}(\mathscr{A} \otimes L)$ as required. Now let $\bar{p} \in \operatorname{Spec}(\mathscr{A} \otimes \overline{k(q)})$ lying over $p \in \operatorname{Spec} \mathscr{A}$. Since $G$ is constant, the geometric stabilizer $H$ of $p$ (that is of $\bar{p}$ ) coincides with the stabilizer of the connected component $\operatorname{Spec}\left((\mathscr{A} \otimes \overline{k(q)})_{\bar{p}}\right)$ and, if we set $\overline{\mathscr{B}}=(\mathscr{A} \otimes \overline{k(q)})_{\bar{p}}$, by 2.6 we get an isomorphism

$$
\mathscr{A} \otimes \overline{k(q)} \simeq \operatorname{ind}_{H}^{G} \overline{\mathscr{B}}
$$


Since $\operatorname{ind}_{H}^{G}: \operatorname{LAlg}_{R}^{H} \longrightarrow \operatorname{LAlg}_{R}^{G}$ is étale, there exists an étale morphism $\operatorname{Spec} B^{\prime} \longrightarrow \operatorname{Spec} B$, $q^{\prime} \in \operatorname{Spec} B^{\prime}$ over $q, \mathscr{B} \in \operatorname{LAlg}^{H} B^{\prime}$ such that $\mathscr{A} \otimes B^{\prime} \simeq \operatorname{ind}_{H}^{G} \mathscr{B}$ and $\mathscr{B} \otimes \overline{k\left(q^{\prime}\right)} \simeq \overline{\mathscr{B}}$. Moreover we have isomorphisms

$$
B^{\prime} \simeq\left(\mathscr{A} \otimes B^{\prime}\right)^{G} \simeq\left(\operatorname{ind}_{H}^{G} \mathscr{B}\right)^{G} \simeq \mathscr{B}^{H}
$$

Thus $\widetilde{\mathscr{A}}=\mathscr{B}$ satisfies the desired conditions.

Lemma 2.8. Let $H$ be an open and closed subgroup of $G, T$ an $R$-scheme and $\mathcal{F} \in \mathrm{QAlg}^{H} T$. Then

where $\mathrm{R}_{H}: \operatorname{Loc}^{G} R \longrightarrow \operatorname{Loc}^{H} R$ is the restriction.

$$
\Omega^{\operatorname{ind}_{H}^{G} \mathcal{F}} \simeq \Omega^{\mathcal{F}} \circ \mathrm{R}_{H}: \operatorname{Loc}^{G} R \longrightarrow \mathrm{QCoh} T
$$

Proof. Given $V \in \operatorname{Loc}^{G} R$ we have

$$
\Omega_{V}^{\operatorname{ind}_{H}^{G} \mathcal{F}}=\operatorname{Hom}^{G}\left(V^{\vee}, \operatorname{ind}_{H}^{G} \mathcal{F}\right) \simeq \operatorname{Hom}^{H}\left(\mathrm{R}_{H}(V)^{\vee}, \mathcal{F}\right)=\Omega_{\mathrm{R}_{H}(V)}^{\mathcal{F}}
$$

\section{REDUCIBILITY OF $G$-Cov FOR NONABELIAN LINEARLY REDUCTIVE GROUPS.}

The aim of this section is to prove the reducibility of $G$-Cov when $G$ is a nonabelian linearly reductive group, that is Theorem $\mathrm{B}$. We fix a base ring $R$ and a finite, flat, finitely presented and linearly reductive group scheme $G$ over $R$.

Definition 3.1. Let $S$ be a scheme and $\mathcal{X}$ be an algebraic stack over $S$. The stack $\mathcal{X}$ is called universally reducible over $S$ if, for all base changes $S^{\prime} \longrightarrow S$, the stack $\mathcal{X} \times{ }_{S} S^{\prime}$ is reducible.

Remark 3.2. It is easy to check that $\mathcal{X}$ is universally reducible over $S$ if and only if for all fields $k$ and maps Spec $k \longrightarrow S$ the fiber is reducible.

We start by stating the generalization of Theorem B we are going to prove at the end of this section.

Theorem 3.3. If $G$ is a finite, flat and finitely presented nonabelian and linearly reductive group scheme over $R$ then $G$-Cov is reducible. If, moreover, $G$ is defined over a connected scheme, then $G$-Cov is also universally reducible.

Note that, if we do not assume that the base Spec $R$ is connected, we can not conclude that $G$-Cov is universally reducible, since one can always take $G$ as disjoint union of $\mu_{2}$ and $S_{3}$ over Spec $\mathbb{Q} \sqcup \operatorname{Spec} \mathbb{Q}$. On the other hand what happens when the base is not connected is clear from the following Proposition.

Proposition 3.4. The locus of $\operatorname{Spec} R$ where $G$ is abelian is open and closed in Spec $R$.

Proof. Denote by $Z$ this locus and set $S=\operatorname{Spec} R$. Topologically, $|Z|$ is closed in $S$, because it is the locus where the maps $G \times G \longrightarrow G$ given by $(g, h) \longmapsto g h$ and $(g, h) \longmapsto h g$ coincide and $G$ is flat and proper. We have to prove that, given an algebraically closed field $k$ and a map Spec $k \stackrel{p}{\longrightarrow} S$ such that $G_{k}=G \times k$ is abelian, there exists a fppf neighborhood of $S$ around $p$ where $G$ is abelian. By [AOV08, Theorem 2.19], we can assume that $G=\Delta \ltimes H$, where $\Delta$ is diagonalizable and $H$ is constant. If $G_{k}$ is abelian, then $H$ is abelian, the map $H \longrightarrow$ Aut $\Delta \simeq \operatorname{Aut}\left(\operatorname{Hom}\left(\Delta, \mathbb{G}_{m}\right)\right)^{o p}$ is trivial and therefore $G \simeq \Delta \times H$ is abelian.

Definition 3.5. We say that an open substack $\mathcal{U}$ of an algebraic stack $\mathcal{X}$ is schematically dense if $\mathcal{X}$ is the only closed substack of $\mathcal{X}$ containing $\mathcal{U}$. If $\mathcal{U}$ is a quasi-compact open substack of $\mathcal{X}$ its schematic closure is the minimum of the closed substacks of $\mathcal{X}$ containing $\mathcal{U}$ or, alternatively, the (unique) closed substack $\mathcal{Z}$ of $\mathcal{X}$ such that $\mathcal{U} \subseteq \mathcal{Z}$ and $\mathcal{U}$ is schematically dense in $\mathcal{Z}$. 
We denote by $\mathcal{Z}_{G}$ the schematic closure of B $G$ inside $G$-Cov and we call it the main irreducible component of $G$-Cov.

The existence of the schematic closure as stated above and the fact that it is stable by flat base changes follows from [Gro66, Theorem 11.10.5]. Although we have called $\mathcal{Z}_{G}$ the main irreducible component of $G$-Cov, the stack $\mathcal{Z}_{G}$ is irreducible if and only if $\operatorname{Spec} R$ is irreducible, because this is the only case in which $\mathrm{B} G$ is irreducible.

Lemma 3.6. Let $H$ be an open and closed subgroup scheme of $G$ and $\mathscr{B} \in \mathrm{LAlg}_{R}^{H}$. Then

$$
\operatorname{ind}_{H}^{G} \mathscr{B} \in \mathrm{B} G \Longleftrightarrow \mathscr{B} \in \mathrm{B} H, \operatorname{ind}_{H}^{G} \mathscr{B} \in \mathcal{Z}_{G} \Longleftrightarrow \mathscr{B} \in \mathcal{Z}_{H}
$$

Proof. The fact that $\mathscr{B} \in \mathrm{B} H \Longrightarrow \operatorname{ind}_{H}^{G} \mathscr{B} \in B G$ is well known. For the converse set $P=\operatorname{Spec} \mathscr{B}$ and consider it as a sheaf of sets over $\mathrm{Sch} / T$ with a right action of $H$, where $T$ is the $R$-scheme over which $\mathscr{B}$ is defined. Then $Q=\operatorname{Spec}\left(\operatorname{ind}_{H}^{G} \mathscr{B}\right)$ is by definition $(P \times G) / H$, where the $H$ action on $P \times G$ is given by $(p, g) h=\left(p h, h^{-1} g\right)$ and the $G$-action is on the right. It is easy to check that the natural map $P \longrightarrow Q, p \longmapsto(p, 1)$ is an $H$-equivariant monomorphism. Assume that $Q$ is a $G$-torsor. It follows that $H$ acts freely on $P$, so that sheaf quotient $P / H$ and stack quotient $[P / H]$ coincide. Moreover $P / H \longrightarrow Q / G$ is an isomorphism, so that $P / H \simeq Q / G \simeq T$ because $Q$ is a $G$-torsor. In conclusion $P \longrightarrow[P / H] \simeq T$ is an $H$-torsor.

Since $H$-Cov (resp. $G$-Cov) is closed in $\operatorname{LAlg}_{R}^{H}$ (resp. $\operatorname{LAlg}_{R}^{G}$ ) by Theorem A, it follows that $\mathcal{Z}_{H}$ (resp. $\mathcal{Z}_{G}$ ) is the schematic closure of B $H$ (resp. B $G$ ) inside $\operatorname{LAlg}_{R}^{H}$ (resp. $\operatorname{LAlg}_{R}^{G}$ ). The second equivalence therefore follows because flat maps preserve schematic closures and ind $H_{H}^{G}: \operatorname{LAlg}_{R}^{H} \longrightarrow$ $\mathrm{LAlg} G$ is étale by 2.1 .

Definition 3.7. Assume that $G$ is a good linearly reductive group and that $\operatorname{Spec} R$ is connected. Given a scheme $T$, we will say that a functor $\Omega: \operatorname{Loc}^{G} R \longrightarrow \operatorname{Loc} T$ (a sheaf of algebras $\mathscr{A} \in$ $\operatorname{LAlg}^{G} T$ ) has equivariant constant rank (or is of equivariant constant rank) if for all $V \in \operatorname{Loc}^{G} R$ the locally free sheaf $\Omega_{V}\left(\Omega_{V}^{\mathscr{A}}=(V \otimes \mathscr{A})^{G}\right)$ has constant rank. In this case we define the rank function $\mathrm{rk}^{\Omega}: I_{G} \longrightarrow \mathbb{N}\left(\mathrm{rk}^{\mathscr{A}}: I_{G} \longrightarrow \mathbb{N}\right)$ as

$$
\mathrm{rk}_{V}^{\Omega}=\mathrm{rk}_{V},\left(\mathrm{rk}_{V}^{\mathscr{A}}=\mathrm{rk}_{V}^{\Omega^{\mathscr{A}}}=\mathrm{rk}(V \otimes \mathscr{A})^{G}\right)
$$

Given $f: I_{G} \longrightarrow \mathbb{N}$ we will still call $f$ the extension $f: \operatorname{Loc}^{G} R \longrightarrow \mathbb{N}$ given by

$$
f_{U}=\sum_{V \in I_{G}} \operatorname{rk}\left(\operatorname{Hom}^{G}(V, U)\right) f_{V}
$$

so that if $\Omega: \operatorname{Loc}^{G} R \longrightarrow \operatorname{Loc} T$ is an $R$-linear functor then $\operatorname{rk}_{V}^{\Omega}=\operatorname{rk}_{V}$ for all $V \in \operatorname{Loc}^{G} R$.

Lemma 3.8. [MM03] A constant group whose proper subgroups are abelian is solvable.

We are ready for the proof of Theorem 3.3.

Proof of Theorem 3.3. If the base scheme is not connected, then clearly $G$-Cov is reducible. By 3.2 and 3.4, we can assume that $S=\operatorname{Spec} k$, where $k$ is a field. Notice that $G$-Cov is reducible if and only if $\mathcal{Z}_{G}(\bar{k}) \subsetneq G$ - $\operatorname{Cov}(\bar{k})$, where $\bar{k}$ is the algebraic closure of $k$. Moreover $\mathcal{Z}_{G \times \bar{k}} \simeq \mathcal{Z}_{G} \times \bar{k}$. Thus, taking into account 3.4, we can assume that $k$ is algebraically closed, so that $G$ is a good linearly reductive nonabelian group scheme.

Let $H$ be an open and closed subgroup of $G$. We claim that if one of the following statement holds then $G$-Cov is reducible:

1) $\mathrm{H}$-Cov is reducible

2) there exists $f: I_{H} \longrightarrow \mathbb{N}$ whose extension $f: \operatorname{Loc}^{H} k \longrightarrow \mathbb{N}$ is such that $f_{\mathrm{R}_{H} V}=\operatorname{rk} V$ for any $V \in I_{G}$ and there exists $\Delta \in I_{H}$ such that $f_{\Delta} \neq \mathrm{rk} \Delta$ 
Assume that $H$-Cov is reducible and, by contradiction, that $G$-Cov is irreducible. If $B \in$ $H$ - $\operatorname{Cov}(k)$ then $\operatorname{ind}_{H}^{G} B \in G$ - $\operatorname{Cov}(k)=\mathcal{Z}_{G}(k)$ and so $B \in \mathcal{Z}_{H}(k)$ by 3.6. Therefore $H$-Cov is irreducible.

Now let $f: I_{H} \longrightarrow \mathbb{N}$ as in 2) and define

$$
F=\bigoplus_{R \neq \Delta \in I_{H}} \Delta^{\vee} \otimes k^{f_{\Delta}}, B=k \oplus F
$$

so that $f=\mathrm{rk}^{B}$ (note that by hypothesis we have $f_{R}=1$ ). Setting $F^{2}=0$ we obtain a structure of algebra on $B$ such that $B \in \operatorname{LAlg}^{H} k$. We claim that $A=\operatorname{ind}_{H}^{G} B \in\left(G\right.$-Cov $\left.(k)-\mathcal{Z}_{G}(k)\right)$. Indeed we have $\Omega^{A}=\Omega^{B} \circ \mathrm{R}_{H}$ by 2.8 , so that

$$
\operatorname{rk} \Omega_{V}^{A}=\operatorname{rk} \Omega_{\mathrm{R}_{H} V}^{B}=f_{\mathrm{R}_{H} V}=\operatorname{rk} V \text { for all } V \in \operatorname{Rep}^{G} R
$$

Thus $\Omega^{A} \in \operatorname{Mon}_{R, \text { reg }}^{G}$ and, since $G$ is good, by Theorem A we can conclude that $A \in G$-Cov. If by contradiction $A \in \mathcal{Z}_{G}(k)$, by 3.6 we have $B \in \mathcal{Z}_{H}(k) \subseteq H$ - $\operatorname{Cov}(k)$ so that, by Theorem A, $\operatorname{rk} \Omega_{\Delta}^{B}=f_{\Delta}=\operatorname{rk} \Delta$ for all $\Delta \in I_{H}$, which is not the case.

We return now to the original statement. We are going to use notation from 2.3. By [AOV08, Theorem 2.19] we have $G=G_{1} \ltimes \underline{G}$ with $G_{1}$ diagonalizable. In particular $\underline{G}$ cannot be trivial. If $\underline{G}$ is not solvable take a minimal nonabelian subgroup $K$ of $\underline{G}$. All the proper subgroups of $K$ are abelian and therefore $K$ is solvable thanks to 3.8. If we call $\phi: G \longrightarrow \underline{G}$ the natural projection, then $G^{\prime}=\phi^{-1}(K)$ is a nonabelian open and closed subgroup of $G$ such that $G^{\prime} \simeq K$ is solvable. Using situation 1) above we can replace $G$ by $G^{\prime}$, that is assume that $\underline{G}$ is solvable. In particular there exists a surjective homomorphism $\alpha: G \longrightarrow \mathbb{Z} / p \mathbb{Z}$ for some prime $p$. Set $H=\operatorname{Ker} \alpha$, which is an open and closed subgroup of $G$. If $H$ is nonabelian, using again situation 1 ) we can replace $G$ by $H$. Proceeding by induction we can finally assume to have a surjection $G \longrightarrow \mathbb{Z} / p \mathbb{Z}$ whose kernel $H$ is abelian. Since $H$ is linearly reductive and $k$ is algebraically closed the group $H$ is diagonalizable. Set $N=\operatorname{Hom}\left(H, \mathbb{G}_{m}\right)$. We will construct an $f: I_{H} \longrightarrow \mathbb{N}$ as in situation 2) above. This will conclude the proof.

Since $H$ is commutative, the group $G / H \simeq \mathbb{Z} / p \mathbb{Z}$ acts on $H$ and on $N=\operatorname{Hom}\left(H, \mathbb{G}_{m}\right)$ by conjugation. Given $m \in N$ we are going to denote by $V_{m}$ the corresponding one-dimensional representation of $H$. Let $\mathcal{R} \subseteq N$ be a set of representatives of $N /(\mathbb{Z} / p \mathbb{Z})$. Note that, since $p$ is prime, an element $n \in N$ is fixed or its orbit $o(n)$ has order $p$. We claim that if $V \in I_{G}$ there exists a unique $m \in \mathcal{R}$ such that

$$
\mathrm{R}_{H} V=V_{m}^{\text {rk } V} \text { with }|o(m)|=1 \text { or } V=\operatorname{ind}_{H}^{G} V_{m} \text { with }|o(m)|=p
$$

Indeed there exists $m \in N$ such that $V \subseteq \operatorname{ind}_{H}^{G} V_{m}$. Given $n, n^{\prime} \in N$ we have

$$
\mathrm{R}_{H} \operatorname{ind}_{H}^{G} V_{n}=\bigoplus_{g \in \mathbb{Z} / p \mathbb{Z}} V_{g(n)} \text { and }\left(\operatorname{ind}_{H}^{G} V_{n} \simeq \operatorname{ind}_{H}^{G} V_{n^{\prime}} \Longleftrightarrow n^{\prime} \in o(n)\right)
$$

So we can assume $m \in \mathcal{R}$. Moreover such an $m$ is unique since if $V \subseteq \operatorname{ind}_{H}^{G} V_{m^{\prime}}, \mathrm{R}_{H} V$ contains some $V_{n}$ where $n \in N$ is in the orbit of both $m$ and $m^{\prime}$. In particular, if $|o(m)|=1$, then $\operatorname{ind}_{H}^{G} V_{m}=V_{m}^{p}$ and therefore $\mathrm{R}_{H} V=V_{m}^{\mathrm{rk} V}$. So assume $|o(m)|=p$. Given $W \in \operatorname{Loc}^{G} k\left(\operatorname{Loc}^{H} k\right)$ and $g \in G(k)$ call $W_{g}$ the representation of $G(H)$ that has $W$ as underlying vector space, while the action of $G(H)$ is given by $t \star x=\left(g^{-1} t g\right) x$. Note that by definition $\left(V_{n}\right)_{g}=V_{g(n)}$. In particular the multiplication by $g^{-1}$ on $V$ yields a $G$-equivariant isomorphism $V \simeq V_{g}$ and therefore $V_{n} \subseteq \mathrm{R}_{H} V$ implies that $V_{g(n)} \subseteq \mathrm{R}_{H} V$. Since $|o(m)|=p$ we can conclude that $V=\operatorname{ind}_{H}^{G} V_{m}$. Define

$$
f_{V_{n}}=\left\{\begin{array}{cc}
|o(n)| & \text { if } n \in \mathcal{R} \\
0 & \text { otherwise }
\end{array}\right.
$$


We claim that $f$ satisfies the property 2). Indeed if $V \in I_{G}$ and there exists $m \in \mathcal{R}$ such that $V=V_{m}^{\text {rk } V}$ with $|o(m)|=1$ then $f_{\mathrm{R}_{H} V}=\operatorname{rk} V f_{V_{m}}=\operatorname{rk} V$. Otherwise there exists $m \in \mathcal{R}$ with $|o(m)|=p$ such that

$$
V=\operatorname{ind}_{H}^{G} V_{m} \Longrightarrow f_{\mathrm{R}_{H} V}=\sum_{g \in \mathbb{Z} / p \mathbb{Z}} f_{V_{g(m)}}=p=\mathrm{rk} V
$$

Finally note that if $n \in \mathcal{R}$ is such that $|o(n)|=p$ then $f_{V_{n}}=p \neq 1=\operatorname{rk} V_{n}$. So we have to show that such an $n$ exists. If by contradiction this is false, then the actions of $\mathbb{Z} / p \mathbb{Z}$ on $N$ and $H$, as well as the action of $G$ on $H$ by conjugation are trivial. So $H$ commutes with all the elements of $G$. Let $g \in G(k) \simeq \underline{G}$ not in $H$, so that it lies over a generator of $G / H \simeq \mathbb{Z} / p \mathbb{Z}$. If $T$ is a $k$-scheme, any element of $G(T)$ can be written as $h g^{i}$ with $h \in H(T)$ and $0 \leq i<p$. It is straightforward to check that two such elements commute and that therefore $G$ is abelian, which is not the case.

\section{Regularity in CODIMENSION 1}

The aim of this section is to prove Theorem C. In this section we fix a finite and étale group scheme $G$ over $R$. We require the étaleness condition on $G$ because we want $G$-torsors to be regular over a regular base.

We start with some definitions and remarks. In what follows $T$ will be an arbitrary $R$-scheme if not specified otherwise.

Remark 4.1. If $f: X \longrightarrow T$ is a cover with an action of $G$ then $f$ is a $G$-torsor if and only if $f$ is étale, $X / G=T$ and $\operatorname{rk} f_{*} \mathcal{O}_{X}=\mathrm{rk} G$. The implication $\Longrightarrow$ is easy. For the converse, since the locus where $f$ is a $G$-torsor is open in $T$ and taking invariants commutes with flat base changes of $T$, we can assume that $T=\operatorname{Spec} B$, where $B$ is a local ring, that $G$ is constant and that $X$ is a disjoint union of $\operatorname{rk} G$ copies of $T$. Since $G$ acts transitively on the closed points of $X$ because $X / G=T$, the orbit map $G \times T \longrightarrow X$ is an étale surjective cover. The rank condition implies that this is an isomorphism.

Remark 4.2. If $G$ is a good linearly reductive group and $V \in I_{G}$ then $\operatorname{rk} V \in R^{*}$ and the evaluation map $e_{V}: V \otimes V^{\vee} \longrightarrow R$ induces an isomorphism $\left(V \otimes V^{\vee}\right)^{G} \longrightarrow R$. By a local check we see that $e_{V}$ is surjective and, since $G$ is linearly reductive, we can conclude that $\left(V \otimes V^{\vee}\right)^{G} \longrightarrow R$ is surjective too. Moreover we have a $G$-equivariant isomorphism $\operatorname{Hom}_{R}(V, V) \simeq V \otimes V^{\vee}$ and the map $e_{V}$ corresponds to the trace map $\operatorname{tr}_{V}: \operatorname{Hom}_{R}(V, V) \longrightarrow R$ under this isomorphism. Since $\operatorname{Hom}_{R}^{G}(V, V)=R \operatorname{Rid}_{V}$ by 1.9 we can conclude that $\left(V \otimes V^{\vee}\right)^{G} \longrightarrow R$ is an isomorphism and, since $\operatorname{tr}_{V}\left(\mathrm{id}_{V}\right)=\operatorname{rk} V$, that $\mathrm{rk} V \in R^{*}$.

Definition 4.3. Let $f: X \longrightarrow T$ be a cover. The trace map of $f$ will be denoted by

$$
\operatorname{tr}_{f}: f_{*} \mathcal{O}_{X} \longrightarrow \mathcal{O}_{T}
$$

We also set

$$
\tilde{\operatorname{tr}}_{f}: f_{*} \mathcal{O}_{X} \longrightarrow\left(f_{*} \mathcal{O}_{X}\right)^{\vee}, x \longmapsto \operatorname{tr}_{f}(x \cdot-) \text { and } \mathcal{Q}_{f}=\operatorname{Coker}\left(\tilde{\operatorname{tr}}_{f}\right) \in \operatorname{QCoh}(T)
$$

The discriminant section $s_{f} \in\left(\operatorname{det} f_{*} \mathcal{O}_{X}\right)^{-2}$ is the section induced by the determinant of the $\operatorname{map} \tilde{\operatorname{tr}}_{f}$.

Assume now that $G$ acts on $X$ over $T$ and that $X / G=T$ and consider $V \in \operatorname{Loc}^{G} R$. If $f$ is a $G$-cover or $G$ is linearly reductive we denote by

$$
\Omega^{f}: \operatorname{Loc}^{G} R \longrightarrow \operatorname{Loc} T, \Omega^{f}=\left(f_{*} \mathcal{O}_{X} \otimes-\right)^{G}
$$


the associated monoidal functor (see Theorem A), by

$$
\omega_{f, V}: \Omega_{V}^{f} \otimes \Omega_{V^{\vee}}^{f} \longrightarrow \Omega_{V \otimes V^{\vee}}^{f} \longrightarrow \Omega_{R}^{f} \simeq \mathcal{O}_{T}
$$

where the first map is given by the monoidality, while the second is induced by the evaluation $e_{V}: V \otimes V^{\vee} \longrightarrow R$, by

$$
\xi_{f, V}: \Omega_{V^{\vee}}^{f} \longrightarrow\left(\Omega_{V}^{f}\right)^{\vee}
$$

the induced map and set $\mathcal{Q}_{f, V}=\operatorname{Coker}\left(\xi_{f, V}\right)$. If $f$ is a $G$-cover, then the source and target of the $\operatorname{map} \xi_{f, V}$ are locally free sheaves of the same rank $\mathrm{rk} V$ by Theorem A, and we denote by

$$
s_{f, V} \in\left(\operatorname{det} \Omega_{V}^{f} \otimes \operatorname{det} \Omega_{V^{\vee}}^{f}\right)^{-1}
$$

the section induced by $\operatorname{det} \xi_{f, V}$.

When $\mathscr{A} \in \operatorname{LAlg}^{G} T$ and $f: \operatorname{Spec} \mathscr{A} \longrightarrow T$ we will use the subscript $-\mathscr{A}$ instead of $-{ }_{f}$.

Remark 4.4. If $\mathscr{A} \in \operatorname{LAlg}^{G} T$ then $\operatorname{tr}_{\mathscr{A}}: \mathscr{A} \longrightarrow \mathcal{O}_{T}$ is $G$-equivariant. Indeed one can assume $T$ is affine, $G$ is constant and $\mathscr{A}$ is free and use the invariancy of the trace map under conjugation.

Lemma 4.5. Assume that $R$ is a local ring, that $G$ is a good linearly reductive group and let $\mathscr{A} \in \operatorname{LAlg}^{G} T$ be such that $\mathscr{A}^{G}=\mathcal{O}_{T}$ and $\operatorname{rk} \mathscr{A}=\operatorname{rk} G$. Then

$$
\operatorname{Ker} \operatorname{tr}_{\mathscr{A}} \simeq \bigoplus_{R \neq V \in I_{G}} V^{\vee} \otimes \Omega_{V}^{\mathscr{A}} \text { and } \mathcal{Q}_{\mathscr{A}} \simeq \bigoplus_{V \in I_{G}} V^{\vee} \otimes \mathcal{Q}_{\mathscr{A}, V}
$$

Moreover if $\mathscr{A} \in G$-Cov then there exists an isomorphism

$$
\left(\operatorname{det} f_{*} \mathcal{O}_{X}\right)^{-2} \simeq \bigotimes_{V \in I_{G}}\left(\operatorname{det}\left(\Omega_{V}^{f}\right)^{-1} \otimes \operatorname{det}\left(\Omega_{V^{\vee}}^{f}\right)^{-1}\right)^{\mathrm{rk} V} \text { such that } s_{f} \longmapsto \bigotimes_{V \in I_{G}} s_{f, V}^{\otimes \mathrm{rk} V}
$$

Proof. Notice that, since $R$ is local, then if $V \in I_{G}$ there exists a unique $\hat{V} \in I_{G}$ such that $\hat{V} \simeq V^{\vee}$. For all $V \in I_{G}$ let us fix an equivariant isomorphism $\zeta_{V}: V^{\vee} \longrightarrow \hat{V}$. For simplicity set also $\Omega=\Omega^{\mathscr{A}}: \operatorname{Loc}^{G} R \longrightarrow \operatorname{Loc} T$.

Since $\operatorname{tr}_{\mathscr{A}}: \mathscr{A} \longrightarrow \mathcal{O}_{T}$ is $G$-invariant, we have that $\operatorname{Ker}_{\mathscr{A}}$ is $G$-invariant too. By 1.17 we have

$$
\operatorname{Ker} \operatorname{tr}_{\mathscr{A}}=\bigoplus_{V \in I_{G}} V^{\vee} \otimes \Gamma_{V} \text { with } \Gamma_{V} \subseteq \Omega_{V}
$$

Since $G$ is linearly reductive and $\operatorname{rk} \mathscr{A}=\operatorname{rk} G$, we have $\operatorname{tr}_{\mathscr{A}}(1) \in \mathcal{O}_{T}^{*}$ and, in particular, that $\operatorname{tr}_{\mathscr{A}}: \mathscr{A} \longrightarrow \mathcal{O}_{T}$ is surjective. So

$$
\mathcal{O}_{T}=\bigoplus_{V \in I_{G}} V^{\vee} \otimes\left(\Omega_{V} / \Gamma_{V}\right)
$$

is a $G$-equivariant decomposition and therefore $\Gamma_{V}=\Omega_{V}$ for $R \neq V \in I_{G}$ and $\Gamma_{R}=0$. In other words $\operatorname{tr}_{\mathscr{A}}=(\operatorname{rk} G) \pi$, where $\pi: \mathscr{A} \longrightarrow \mathcal{O}_{T}$ is the projection according to the $G$-equivariant decomposition of $\mathscr{A}$. We are going to use the description given in 1.17 of the product of

$$
\mathscr{A}=\bigoplus_{V \in I_{G}} V^{\vee} \otimes \Omega_{V}
$$

using the maps $\alpha_{U}: U^{\vee} \otimes \Omega_{U} \longrightarrow \mathscr{A}$ for $U \in \operatorname{Loc}^{G} R$. Notice that, given $V, W \in I_{G}$, the product of elements of $V^{\vee} \otimes \Omega_{V}$ and $W^{\vee} \otimes \Omega_{W}$ lies in $\operatorname{Ker} \operatorname{tr}_{\mathscr{A}}=\operatorname{ker} \pi$, i.e. has no component in $\mathscr{A}^{G} \simeq R^{\vee} \otimes \Omega_{R}$, except for the case $(V \otimes W)^{G} \neq 0$. Since

$$
(V \otimes W)^{G}=\operatorname{Hom}^{G}\left(V, W^{\vee}\right)
$$

this is the case only when $W=\hat{V}$. So the trace map $\tilde{\operatorname{tr}}_{\mathscr{A}}: \mathscr{A} \longrightarrow \mathscr{A}^{\vee}$ is the direct sum of the maps

$$
\bar{\xi}_{V}: V^{\vee} \otimes \Omega_{V} \longrightarrow\left((\hat{V})^{\vee} \otimes \Omega_{\hat{V}}\right)^{\vee}
$$


induced by $\delta_{V}: V^{\vee} \otimes \Omega_{V} \otimes(\hat{V})^{\vee} \otimes \Omega_{\hat{V}} \longrightarrow \mathscr{A} \otimes \mathscr{A} \longrightarrow \mathscr{A} \stackrel{\operatorname{tr}_{\mathscr{A}}}{\longrightarrow} \mathcal{O}_{T}$, which is also the composition

$$
V^{\vee} \otimes \Omega_{V} \otimes(\hat{V})^{\vee} \otimes \Omega_{\hat{V}} \simeq(V \otimes \hat{V})^{\vee} \otimes \Omega_{V} \otimes \Omega_{\hat{V}} \longrightarrow(V \otimes \hat{V})^{\vee} \otimes \Omega_{V \otimes \hat{V}} \stackrel{\alpha_{V \otimes \hat{V}}}{\longrightarrow} \mathscr{A} \stackrel{\mathrm{rk} G \pi}{\longrightarrow} \mathcal{O}_{T}
$$

Denote by $e_{V}: V \otimes V^{\vee} \longrightarrow R$ the evaluation map. By replacing $\hat{V}$ by $V^{\vee}$ using the given isomorphism, we are going to check that the composition of the last two maps above is the evaluation $\left(V \otimes V^{\vee}\right)^{\vee} \simeq V^{\vee} \otimes V \stackrel{e_{V}}{\longrightarrow} R$ tensor $\Omega_{e_{V}}$, up to an invertible element. This will imply that $\bar{\xi}_{V}$ is isomorphic to the map

$$
\operatorname{id}_{V^{\vee}} \otimes \xi_{\mathscr{A}, V^{\vee}}: V^{\vee} \otimes \Omega_{V} \longrightarrow V^{\vee} \otimes\left(\Omega_{V^{\vee}}\right)^{\vee}
$$

and, from this, the claimed result easily follow.

By 4.2 the map $e_{V}: V \otimes V^{\vee} \longrightarrow R$ is surjective and it extends to a $G$-equivariant isomorphism $\gamma: V \otimes V^{\vee} \longrightarrow R \oplus Z$ where $Z \in \operatorname{Loc}^{G} R$ is such that $Z^{G}=0$. By 1.17 we have that $\alpha_{V \otimes V^{\vee}}=$ $\alpha_{R \oplus Z} \circ\left(\left(\gamma^{\vee}\right)^{-1} \otimes \Omega_{\gamma}\right)$ and, since $Z^{G}=0$, that $\pi \circ \alpha_{R \oplus Z}:(R \oplus Z)^{\vee} \otimes \Omega_{R \oplus Z} \longrightarrow \mathcal{O}_{T} \simeq R^{\vee} \otimes \Omega_{R}$ is the tensor product of the two natural projections. Since $V \otimes V^{\vee} \stackrel{\gamma}{\longrightarrow} R \oplus Z \longrightarrow R$ is $e_{V}$, we can conclude that $\pi \circ \alpha_{V \otimes V^{\vee}}$ is the tensor product of $\Omega_{e_{V}}: V \otimes V^{\vee} \longrightarrow R$ and $\left(V \otimes V^{\vee}\right)^{\vee} \stackrel{\left(\gamma^{\vee}\right)^{-1}}{\longrightarrow}$ $(R \oplus Z)^{\vee} \longrightarrow R^{\vee}$. This last map is surjective, $G$-equivariant and therefore it is, up to an invertible element of $R$, the map $\left(V \otimes V^{\vee}\right)^{\vee} \simeq V^{\vee} \otimes V \stackrel{e_{V}}{\longrightarrow} R$ by 4.2 .

Proof of Theorem $C$. Recall that the loci in $Y$ where $f: X \longrightarrow Y$ is a $G$-torsor or a $G$-cover are open thanks to 1.5 and that, when $G$ is constant, it acts transitively on the set of points of $X$ over a given point of $Y$ because $X / G=Y$. In particular the geometric stabilizers of two points of $X$ over a given point of $Y$ are conjugates in $G$ and therefore isomorphic. We start by proving how to deduce the two claims after 3). For the first claim, by 3) we have $\operatorname{rk} f=\operatorname{rk} G / r k T$, so that $f$ is generically a $G$-torsor (that is $T=0$ ) if and only if $\operatorname{rk} f=\operatorname{rk} G$. Moreover when $T=0$ the description of the geometric stabilizers of the codimension 1 points of $X$ over $q$ is contained in 3). For the second claim it is enough to note that the generic fiber of $X$ is $\operatorname{Spec} L$, where $L / k(R)$ is a finite field extension with $L^{G}=k(R)$ and the action of $G$ on $L$ is faithful because Aut $_{Y} X \longrightarrow \operatorname{Aut}_{k(R)} L$ is injective: it follows that $L / k(R)$ is a Galois extension with group $G$ and therefore $\operatorname{rk} f=\operatorname{dim}_{k(R)} L=\operatorname{rk} G$.

We start by showing the equivalence between 1), 2), 3) and the following condition:

$\left.2^{\prime}\right)$ the module $\mathcal{Q}_{f} \otimes \mathcal{O}_{Y, q}$ is defined over $k(q)$ and the integer $\operatorname{rk} H / \operatorname{rk} T$, where $H$ and $T$ are the geometric stabilizers of a point of $X$ over $q$ and a generic point of $X$ respectively, is coprime with $\operatorname{char} k(q)$.

We will show that the quotient $\operatorname{rk} H / \operatorname{rk} T$ is an integer. We are going to use some results and definitions from [Ton15]. In particular all points of $X$ over $q$ are tame with separable residue fields if and only if the common rank (over $\overline{k(q)}$ ) of a connected component of $X \times_{Y} \overline{k(q)}$ is coprime with $\operatorname{char} k(q)$ (see [Ton15, Lemma 1.6, Corollary 1.7]). In particular 3) $\Longrightarrow 1$ ): this common rank is $\operatorname{rk} \mathscr{B}=\operatorname{rk} H / \operatorname{rk} T$ applying 2.7 to $\mathscr{B} \otimes \overline{k(q)} / \overline{k(q)}$. Moreover we can replace $Y$ by any étale neighborhood around $q$ and, in particular, assume $G$ constant and $Y=\operatorname{Spec} R$.

Write $X=\operatorname{Spec} \mathscr{A}$ with $\mathscr{A} \in \operatorname{LAlg}^{G} R$ and let $H$ be the geometric stabilizer of a point of Spec $\mathscr{A}$ over $q$. By 2.7 we can assume $\mathscr{A} \simeq \operatorname{ind}_{H}^{G} \widetilde{\mathscr{A}}$ with $\widetilde{\mathscr{A}} \in \mathrm{LAlg}^{H} R$ such that $\widetilde{\mathscr{A}} \otimes_{R} \overline{k(q)}$ is local, $\widetilde{\mathscr{A}^{H}}=R$ and $H$ is the geometric stabilizer of the maximal ideal of $\widetilde{\mathscr{A}} \otimes_{R} R_{q}$. As rings we have $\mathscr{A} \simeq \widetilde{\mathscr{A}}^{(\mathrm{rk} G / \mathrm{rk} H)}$, so that $\mathcal{Q}_{\mathscr{A}} \simeq \mathcal{Q}_{\widetilde{\mathscr{A}}}^{(\mathrm{rk} G / \mathrm{rk} H)}, s_{\mathscr{A}} \simeq s_{\widetilde{A}}^{(\mathrm{rk} G / \mathrm{rk} H)}$ and $\mathscr{A}$ is regular in the points over $q$ if and only if the local ring $\mathscr{A} \otimes_{R} R_{q}$ is regular. The above discussion shows that we can assume that $\mathscr{A} \otimes_{R} \overline{k(q)}$ is local and that $G$ is its geometric stabilizer. Let $\bar{G}$ be the image of the map $G \longrightarrow$ Aut $\mathscr{A}$ and note that all the maps Aut $\mathscr{A} \longrightarrow \operatorname{Aut}\left(\mathscr{A} \otimes R_{q}\right) \longrightarrow \operatorname{Aut}(\mathscr{A} \otimes k(R))$ are injective because $\mathscr{A}$ is a locally free $R$-module. The equivalence between 1),2) and $2^{\prime}$ ) can 
be checked directly on $R_{q}$. Since being a $\bar{G}$-cover is an open condition, also 1) $\Longrightarrow 3$ ) can be checked on $R_{q}$. Thus we can assume that $R$ is a DVR (discrete valuation ring), so that $\mathscr{A}$ is also a local ring.

Notice that 2),3) and $2^{\prime}$ ) implies that $\mathscr{A} / R$ is generically étale. This also follows from 1$)$ : if $\mathscr{A}$ is a domain then $\mathscr{A} \otimes k(R)$ is a field extension of $k(R)$ with $(\mathscr{A} \otimes k(R))^{G}=k(R)$ and therefore separable. Thus we can assume that $\mathscr{A} / R$ is generically étale so that, by [Ton15, Corollary 1.7], it follows that $\mathscr{A} / R$ is tame with separable residue fields if and only if rk $\mathscr{A}$ and char $k(q)$ are coprime. Since $G$ acts transitively on $Z=\operatorname{Spec}(\mathscr{A} \otimes \overline{k(R)})$, it follows that $Z \simeq G / T$ as $G$-space, where $T$ is the geometric stabilizer of a generic point of $\mathscr{A}$. In particular $\operatorname{rk} \mathscr{A}=\operatorname{rk} G / \operatorname{rk} T$, which is an integer. Thus [Ton15, Main Theorem] exactly implies the equivalence between the conditions 1), 2) and $2^{\prime}$ ).

It remains to show 1$) \Longrightarrow 3$ ). Since $\mathscr{A}$ is a domain, $\mathscr{A} \otimes k(R)$ is a field. Moreover $\bar{G}$ acts faithfully on $\mathscr{A} \otimes k(R)$ and $(\mathscr{A} \otimes k(R))^{\bar{G}}=k(R)$. It follows that $\mathscr{A} \otimes k(R) / k(R)$ is a Galois extension with group $\bar{G}$ and therefore a $\bar{G}$ torsor. It follows that $\operatorname{Ker}(G \longrightarrow \bar{G})=T$ is the geometric stabilizer of the generic point of $\mathscr{A}$. In particular $\operatorname{rk} \bar{G}$ is coprime with char $k(q)$, which implies that the map $\bar{G} \longrightarrow$ Aut $\mathscr{A} \longrightarrow$ Aut $\left(p / p^{2}\right) \simeq k(p)^{*}$, where $p$ is the maximal ideal of $\mathscr{A}$, is injective and therefore that $\bar{G}$ is cyclic. Thus $\bar{G}$ is linearly reductive over $R$ and, since $\bar{G}$-Cov $\subseteq \operatorname{LAlg}_{R}^{\bar{G}}$ is closed in this case by Theorem A and $\mathscr{A} / R$ is generically a $\bar{G}$-torsor, we can conclude that $\mathscr{A}$ is a $\bar{G}$-cover over $R$.

We now deal with the last part of the statement. In particular we assume from now on that $G$ is linearly reductive and $\operatorname{rk} f=\operatorname{rk} G$. Since 1 ) implies that $f$ is a $G$-cover, more precisely $f \in \mathcal{Z}_{G}(Y)$, we will assume $f \in G$ - $\operatorname{Cov}(Y)$ in what follows.

Denote by $B_{q}$ the strict Henselization of $\mathcal{O}_{Y, q}$, which is an unramified extension of $\mathcal{O}_{Y, q}$ and a DVR, and by $f_{q} \in G-\operatorname{Cov}\left(B_{q}\right)$ the base change of $f$. By 1.16 the group $G_{q}=G \times B_{q}$ has a good representation theory over $B_{q}$. Moreover, if $U, W \in \operatorname{Rep}^{G} R$, then $\xi_{f, U \oplus W}=\xi_{f, U} \oplus \xi_{f, W}$, so that $\mathcal{Q}_{f, U \oplus W} \simeq \mathcal{Q}_{f, U} \oplus \mathcal{Q}_{f, W}$ and everything commutes with base change. Using 4.5 we obtain

$$
\mathcal{Q}_{f} \otimes B_{q} \simeq \bigoplus_{V \in I_{G_{q}}} V^{\vee} \otimes \mathcal{Q}_{f_{q}, V} \simeq \mathcal{Q}_{f, R[G]} \otimes B_{q}
$$

Since for all $U \in \operatorname{Rep}^{G} R$ the representation $U \otimes B_{q}$ splits as a direct sum of representations in $I_{G_{q}}$ we can conclude that 5$) \Longleftrightarrow 2^{\prime}$ ).

Now notice that, for all $U \in \operatorname{Rep}^{G} R$, the number $v_{q}\left(s_{f, U}\right)$ coincides with the length of $\mathcal{Q}_{f, U} \otimes B_{q}$ over $B_{q}$. In particular, for all $U \in \operatorname{Rep}^{G} R$, if $\mathcal{Q}_{f, U} \otimes B_{q}$ is defined over $k(q)$ then $v_{q}\left(s_{f, U}\right) \leq \operatorname{rk}_{q} U$ because $\mathcal{Q}_{f, U} \otimes B_{q}$ is a quotient of $\left(\Omega_{U}^{f}\right)^{\vee} \otimes B_{q}$ which has $\operatorname{rank} \operatorname{rk}_{q} U$. Moreover $\xi_{f, R}$ is by construction an isomorphism so that, if $U \in \operatorname{Loc}^{G} R$, we have $\mathcal{Q}_{f, U}=\mathcal{Q}_{f, U / U^{G}}$ and $v_{q}\left(s_{f, U}\right)=v_{q}\left(s_{f, U / U^{G}}\right)$ because $U \simeq U^{G} \oplus U / U^{G}$. Thus 5) $\left.\Longrightarrow 4\right)$. Since we have

$$
v_{q}\left(s_{f, R[G]}\right)=v_{q}\left(s_{f}\right)=\sum_{V \in I_{G_{q}}} \operatorname{rk} V \cdot v_{q}\left(s_{f_{q}, V}\right) \text { and } v_{q}\left(s_{f, R}\right)=0
$$

we can also conclude that 4$) \Longrightarrow 2$ ).

\section{REFERENCES}

[AOV08] Dan Abramovich, Martin Olsson, and Angelo Vistoli, Tame stacks in positive characteristic, Annales de l'Institut Fourier 58 (2008), no. 4, 1057-1091.

[DM82] Pierre Deligne and James S. Milne, Tannakian Categories, Hodge cycles, motives, and Shimura varieties, Lecture Notes in Mathematics, vol. 900, Springer-Verlag, Berlin, 1982, pp. 101-228.

[Gir71] Jean Giraud, Cohomologie non abélienne, Springer-Verlag, Berlin, 1971. 
[Gro66] Alexander Grothendieck, EGA4-3 - Étude locale des schémas et des morphismes de schémas (Troisiém partie) - Éléments de géométrie algébrique (rédigés avec la collaboration de Jean Dieudonné), 28 ed., Institut des Hautes Études Scientifiques. Publications Mathématiques, 1966.

[Jan87] Jens Carsten Jantzen, Representations of algebraic groups, Pure and Applied Mathematics, vol. 131, Academic Press Inc., 1987.

[Mat89] Hideyuki Matsumura, Commutative ring theory. Translated from the Japanese by Miles Reid, Cambridge University Press, 1989.

[MBL99] Laurent Moret-Bailly and Gerard Laumon, Champs algébriques, first ed., Springer, 1999.

[MM03] George Abram Miller and Halcott Cadwalader Moreno, Non-Abelian Groups in Which Every Subgroup is Abelian, Transactions of the American Mathematical Society 4 (1903), no. 4, 398.

[Sch13] Daniel Schäppi, The formal theory of Tannaka duality, Astérisque 357 (2013), viii +140.

[Ton13a] Fabio Tonini, Stacks of Ramified Covers Under Diagonalizable Group Schemes, International Mathematics Research Notices (2013), 80.

[Ton13b] Stacks of ramified Galois covers, Ph.D. thesis (2013), 192.

[Ton14] _ Sheafification functors and Tannaka's reconstruction, arXiv:1409.4073 (2014), 35.

[Ton15] Trace map and regularity of finite extensions of a DVR, arXiv:1506.04264 (2015), 8.

(Tonini) Freie Universität Berlin, FB Mathematik und Informatik, Arnimallee 3, Zimmer 112A, 14195 Berlin, Deutschland

E-mail address: tonini@zedat.fu-berlin.de 